\title{
The lung microenvironment shapes a dysfunctional response of alveolar macrophages in aging
}

\author{
Alexandra C. McQuattie-Pimentel, ${ }^{1}$ Ziyou Ren, ${ }^{1}$ Nikita Joshi, ${ }^{1}$ Satoshi Watanabe, ${ }^{1}$ Thomas Stoeger, ${ }^{2}$ Monica Chi, ${ }^{1}$ Ziyan Lu, ${ }^{1}$ \\ Lango Sichizya, ${ }^{1}$ Raul Piseaux Aillon, ${ }^{1}$ Ching-I Chen, ${ }^{1}$ Saul Soberanes, ${ }^{1}$ Zhangying Chen, ${ }^{1}$ Paul A. Reyfman, ${ }^{1}$ James M. Walter, ${ }^{1}$ \\ Kishore R. Anekalla, ${ }^{1}$ Jennifer M. Davis, ${ }^{1}$ Kathryn A. Helmin, ${ }^{1}$ Constance E. Runyan, ${ }^{1}$ Hiam Abdala-Valencia, ${ }^{1}$ Kiwon Nam, ${ }^{1}$ \\ Angelo Y. Meliton, ${ }^{3}$ Deborah R. Winter, ${ }^{4}$ Richard I. Morimoto, ${ }^{5}$ Gökhan M. Mutlu, ${ }^{3}$ Ankit Bharat, ${ }^{6}$ Harris Perlman, ${ }^{4}$ \\ Cara J. Gottardi, ${ }^{1}$ Karen M. Ridge, ${ }^{1}$ Navdeep S. Chandel, ${ }^{1}$ Jacob I. Sznajder, ${ }^{1}$ William E. Balch, ${ }^{7}$ Benjamin D. Singer, ${ }^{1,5}$ \\ Alexander V. Misharin, ${ }^{1}$ and G.R. Scott Budinger ${ }^{1}$ \\ 'Department of Medicine, Division of Pulmonary and Critical Care Medicine, Northwestern University, Chicago, Illinois, USA. Department of Chemical and Biological Engineering, Northwestern University, \\ Evanston, Illinois, USA. ${ }^{3}$ Department of Medicine, Division of Pulmonary and Critical Care Medicine, University of Chicago Hospitals, Chicago, Illinois, USA. ${ }^{4}$ Department of Medicine, Division of \\ Rheumatology, Northwestern University, Chicago, Illinois, USA. ${ }^{5}$ Department of Biochemistry and Molecular Genetics, Northwestern University, Evanston, Illinois, USA. ${ }^{6}$ Department of Surgery, Division of \\ Thoracic Surgery, Northwestern University, Chicago, Illinois, USA. ${ }^{7}$ The Scripps Research Institute Department of Chemical Physiology, La Jolla, California, USA.
}

\begin{abstract}
Alveolar macrophages orchestrate the response to viral infections. Age-related changes in these cells may underlie the differential severity of pneumonia in older patients. We performed an integrated analysis of single-cell RNA-Seq data that revealed homogenous age-related changes in the alveolar macrophage transcriptome in humans and mice. Using genetic lineage tracing with sequential injury, heterochronic adoptive transfer, and parabiosis, we found that the lung microenvironment drove an age-related resistance of alveolar macrophages to proliferation that persisted during influenza A viral infection. Ligand-receptor pair analysis localized these changes to the extracellular matrix, where hyaluronan was increased in aged animals and altered the proliferative response of bone marrow-derived macrophages to granulocyte macrophage colony-stimulating factor (CM-CSF). Our findings suggest that strategies targeting the aging lung microenvironment will be necessary to restore alveolar macrophage function in aging.
\end{abstract}

\section{Introduction}

Advanced age is the most important risk factor for severe disease or death in patients with viral pneumonia, including coronavirus disease 2019 (COVID-19) (1, 2). Alveolar macrophages play central roles in lung homeostasis and in the response to airborne environmental toxins and pathogens, including respiratory viruses (3). Careful lineage-tracing studies in mice revealed alveolar macrophages as a long-lived, self-renewing resident lung cell population derived from the fetal liver during embryogenesis (4-6). Epigenomic profiling of alveolar macrophages suggests that their developmental phenotype and function are driven by signals arising from the lung microenvironment (7). In response to viral or other forms of injury, tissue-resident alveolar macrophages (TRAMs) are replaced by monocyte-derived alveolar macrophages (MoAMs) that persist after injury resolves (8). Together, these lines of evidence suggest 3 nonexclusive mechanisms that might explain changes in alveolar macrophages with aging. First, the self-replicating population of TRAMs might undergo epigenetic or other cell-autonomous changes with advancing age. This

Authorship note: ACMP, ZR, AVM, and GRSB contributed equally to this work. Conflict of interest: The authors have declared that no conflict of interest exists. Copyright: () 2021, American Society for Clinical Investigation.

Submitted: May 18, 2020; Accepted: December 9, 2020; Published: February 15, 2021. Reference information: J Clin Invest. 2021;131(4):e140299.

https://doi.org/10.1172/JCl140299. mechanism would be akin to the DNA methylation changes that reproducibly develop in aging $\mathrm{T}$ cells, which persist after adoptive transfer into an age-mismatched host $(9,10)$. Alternatively, or in addition, age-related changes in the lung microenvironment might disrupt normal signals necessary to maintain the function of alveolar macrophages. Finally, repeated injuries over the lifespan could result in the replacement of TRAMs with MoAMs, and differences between these ontologically distinct cell populations might alter the response to environmental challenge (11).

We used a combination of approaches to address these questions in aging mice and validated our findings in aging humans. In both mice and humans, we observed that advanced age was associated with the downregulation of cell-cycle genes in alveolar macrophages. Correspondingly, the number of alveolar macrophages was reduced in old compared with young adult animals. Single-cell RNA-Seq analysis of mice and humans suggested these changes were not attributable to the emergence of an alveolar macrophage subpopulation during aging. Surprisingly, agerelated changes in the transcriptome of TRAMs were largely reversed by heterochronic adoptive transfer, but unaffected by heterochronic parabiosis. Furthermore, the transcriptomic responses of TRAMs and MoAMs were similar during aging and in response to influenza A or bleomycin challenge.

These findings suggest that changes in the transcriptomic identity of alveolar macrophages with advancing age are almost entirely attributable to the lung microenvironment. Analysis 
of ligand-receptor pairs between genes that changed with age in alveolar type 2 (AT2) cells and alveolar macrophages implicated changes in the extracellular matrix in an acquired agerelated hyporesponsiveness to granulocyte macrophage colonystimulating factor (GM-CSF) in alveolar macrophages. Supportive of this hypothesis, the levels of hyaluronan, the ligand for CD44 expressed on alveolar macrophages, were increased in the epithelial lining fluid from old mice compared with that from young mice. Hyaluronan inhibited the proliferation of bone marrow-derived macrophages (BMDMs) cultured on matrix proteins found in the epithelial lining fluid. Collectively, our results implicate age-related changes in the alveolar environment, including changes in the extracellular lining fluid, in the reduced number and altered function of alveolar macrophages in aging. Targeting the lung microenvironment will likely be necessary to restore youthful responses to viral and other inhaled challenges in older individuals.

\section{Results}

All transcriptomic data can be explored online at https://www. nupulmonary.org/mcquattie-pimentel-ren-et-al-2020/.

Advanced age is associated with reduced expression of cell-cycle genes in mice and humans. We flow-sorted TRAMs and AT2 cells from single-cell suspensions of lung tissue from naive young adult (4-6 months old) and old (18-24 months old) mice (Figure 1A; for the AT2 gating strategy and validation in transgenic mice, see Supplemental Figure 1A; supplemental material available online with this article; https://doi.org/10.1172/JCI140299DS1). We found significant differences in the transcriptomes of both TRAMs and AT2 cells from old compared with young adult animals (Figure 1, B-D). These changes were similar in multiple independent cohorts of mice and overlapped significantly with transcriptomic changes in aging alveolar macrophages reported by another group (Supplemental Figure 1, B and C, and ref. 12). Gene Ontogeny (GO) analysis showed that genes related to the "cell cycle" and "translation" were downregulated in old compared with young adult mice, whereas genes involved in the "inflammatory response" were upregulated (Figure 1C). In AT2 cells from old versus young adult mice, we observed a downregulation of genes involved in "cell adhesion" and "extracellular matrix organization," whereas genes involved in the "immune response" and "regulation of biological quality" were upregulated (Figure 1D). These changes persisted in TRAMs and AT2 cells harvested from either young adult or old mice 4 days after infection with the influenza A virus (Figure 1B and Supplemental Figure 1D).

We used single-cell RNA-Seq to determine whether age-related transcriptomic changes in bulk alveolar macrophages or AT2 cells were driven by the emergence of a transcriptionally distinct population of cells. Clustering of alveolar macrophages from 4-, 12-, and 18-month old mice revealed only 2 clusters, both proportionately represented by mice of all ages (Figure 1, E and F). Cluster 2 was characterized by the expression of cell-cycle genes (Mki67 and Top2a), suggesting that it represents actively dividing cells (Supplemental Figure 1E). We did not identify a distinct cluster of alveolar macrophages in old compared with young adult mice (Figure 1G and Supplemental Table 2). The reduced expression of cell-cycle genes in alveolar macrophages from old mice prompted us to quantify alveolar macrophages in lung homogenates from young adult and old mice. We performed a meta-analysis of 6 independent experiments, which revealed a difference of -0.997 (CI -1.662, $-0.170, P=0.016) \times 10^{4}$ alveolar macrophages per lung in old compared with young adult mice (Figure $1 \mathrm{H})$. Alveolar macrophages interact with AT2 and AT1 cells, relying on GM-CSF from AT2 cells for their maintenance $(13,14)$. Hence, we performed a similar analysis of AT 2 cells, in which we observed only 2 clusters of cells, each of which was proportionally represented by lungs of mice from all 3 ages (Figure 1, I-K). One cluster (cluster 2) was characterized by increased expression of $L y z 1$ (Supplemental Figure $1 F)$ and has been noted in previous data sets $(15,16)$. Similar to TRAMs, a distinct subcluster of AT2 cells did not emerge during aging (Figure 1K and Supplemental Figure 1F).

To determine whether analogous age-related transcriptomic changes in alveolar macrophages are found in humans, we integrated macrophage populations from 6 published single-cell RNA-Seq studies conducted on healthy human lung tissue (17-22). Combined, these data included normal lung tissue from 38 subjects over an age range of 17 to 88 (Figure 2, A and B). We identified alveolar macrophages in these data sets by their expression of FABP4 (Figure 2C). Although we observed several clusters of alveolar macrophages in the lung, representation as a function of age was similar across clusters, arguing against the emergence of a transcriptionally distinct population of alveolar macrophages in older people (Supplemental Figure 2, A-D). Accordingly, we generated a pseudo-bulk RNA-Seq transcriptome from each subject (Figure 2, C and D). Examination of alveolar macrophages from individuals younger than 30 years of age compared with those over 60 years of age identified 289 genes whose expression was increased and 677 genes whose expression was reduced in aged compared with young individuals (Figure 2D). GO biological processes that were downregulated with advanced age included "regulation of cell adhesion" and "regulation of cell proliferation." GO biological processes that were elevated in older individuals included the "immune effector process," "positive regulation of tumor necrosis factor production," and "cell activation." These processes were reminiscent of those observed in mice. Consistent with this hypothesis, GSEA using homologs of genes that were differentially expressed in mice showed significant enrichment in human macrophages from older individuals (normalized enrichment score: $1.22 ; P<0.01)$. As with alveolar macrophages, we observed transcriptomic heterogeneity of AT2 cells, but this did not vary as a function of age (Supplemental Figure 2, E-H). Collectively, these results suggest that alveolar macrophages from humans exhibit transcriptomic changes with aging with significant homology to mice and argue against the emergence or loss of a transcriptionally distinct alveolar macrophage subpopulation in aging.

Changes in TRAMs with aging are not cell autonomous. T cells develop predictable changes in their DNA methylome with aging, and these changes persist after heterochronic adoptive transfer $(9,10)$. To determine whether a similar cell-autonomous mechanism drives transcriptomic changes in alveolar macrophages with advancing age, we performed heterochronic adoptive transfer of TRAMs using young adult and old mice as donors and recipients and analyzed alveolar macrophage transcriptomes using RNA- 
A

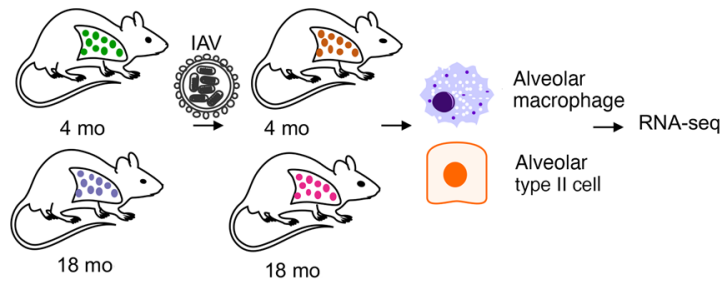

C

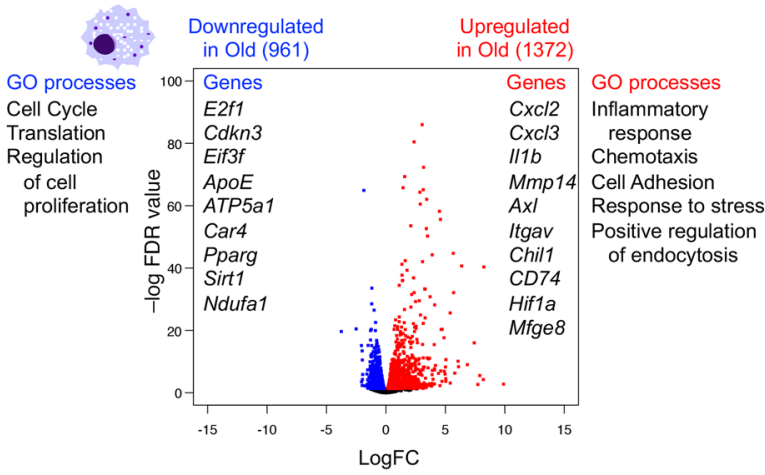

E

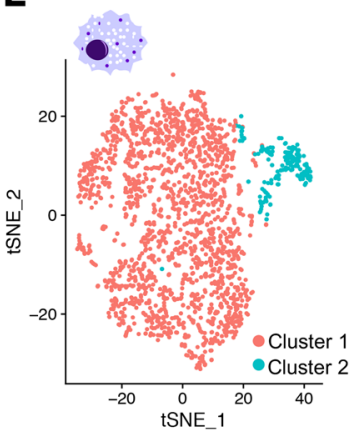

I

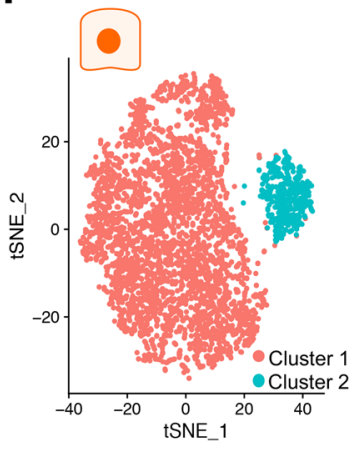

B
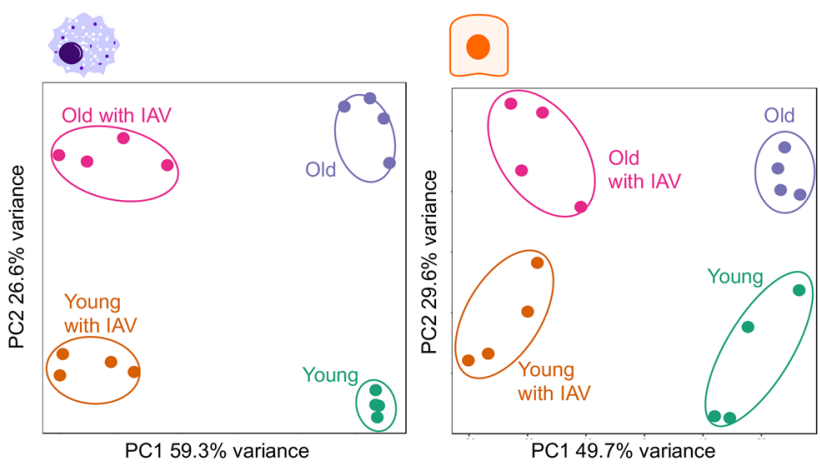

D

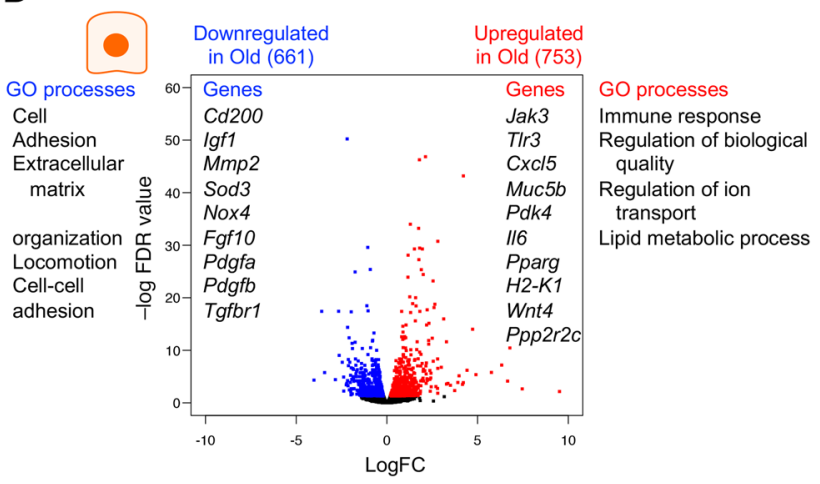

G

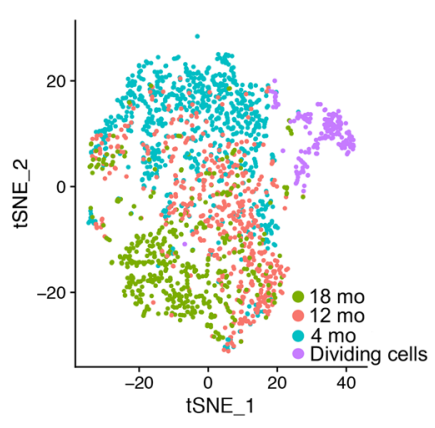

K

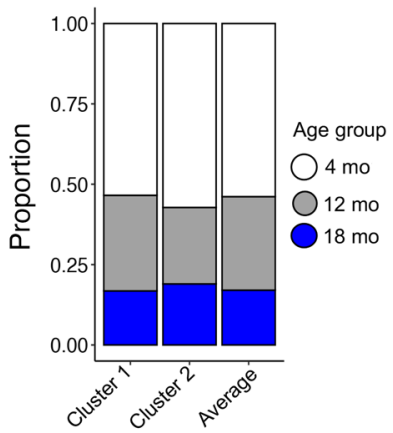

H

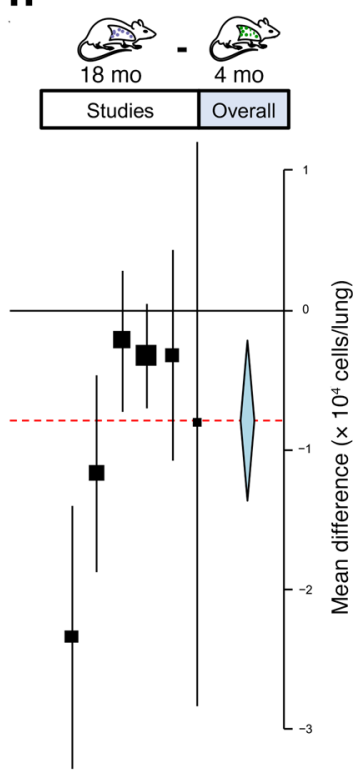

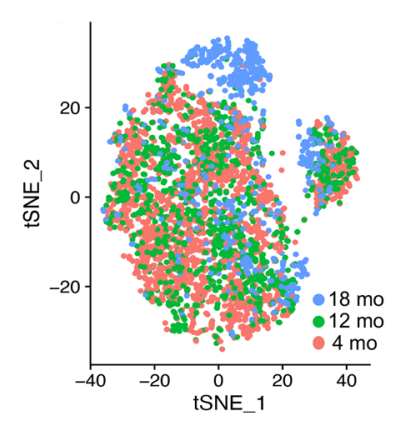


Figure 1. Age-related changes in alveolar macrophage transcriptomes persist during influenza $\mathbf{A}$ infection in mice. (A) Schematic of the experimental design. (B) PCA plots show changes in alveolar macrophage and $A T 2$ cell transcriptomes in response to influenza $A$ infection (PC1) and age (PC2). IAV, influenza $A$ virus. (C) Differentially expressed genes (FDR $q<0.05$ ) between alveolar macrophages from young adult and old mice are shown with the representative genes and GO biological processes (see Supplemental Figure $1 \mathrm{C}$ and Supplemental Table 1 for genes and $\mathrm{GO}$ processes). (D) Differentially expressed genes (FDR $q<0.05$ ) between AT2 cells from young adult and old mice are shown with the representative genes and $\mathrm{CO}$ biological processes (see Supplemental Figure $1 \mathrm{C}$ and Supplemental Table 1 for genes and GO processes. (E) The $t$-distributed stochastic neighbor embedding ( $t S N E$ ) plot shows clusters of alveolar macrophages from 4-, 12-, and 18-month-old mice (see Supplemental Table 2 for the complete list of cluster markers). $n=2$ mice per age group. (F) Bar plot shows the distribution of alveolar macrophages in each cluster as a function of age. (G) tSNE plot shows alveolar macrophages colored by mouse age. (H) Meta-analysis of 6 independent experiments in which alveolar macrophages from lung homogenates from young adult and old mice were quantified by flow cytometry. (I) tSNE plot shows clusters of AT2 cells in single-cell RNA-Seq analysis of lung homogenates from 4-, 12-, and 18-month-old mice. $n=2$ mice per age group. (J) Bar plot shows the distribution of AT2 cells in each cluster as a function of age. (K) $t$ SNE plot shows AT2 cells colored by mouse age. FC, fold change.

Seq (Figure 3A and Supplemental Figure 3, A-C). Attempts to perform intratracheal adoptive transfer of mature TRAMs into mice without depleting the niche with intratracheal liposomal clodronate pretreatment resulted in extremely poor engraftment $(<5 \%$, Supplemental Figure 3, B-D). However, administration of intratracheal liposomal clodronate before adoptive transfer allowed for engraftment rates up to $30 \%$, without the recruitment of neutrophils or other inflammatory cells to the lung (Supplemental Figure 3, B-D). The rate of engraftment of young TRAMs into old mice was significantly lower than the rate of engraftment of old TRAMs into young adult mice, suggesting a loss of proliferative or survival signals in aged animals (Figure 3, B and C). The overall structure of the transcriptomic data visualized by k-means clustering suggested that heterochronic adoptive transfer altered gene expression in adoptively transferred TRAMs toward the age of the recipient (Figure 3, D and E). Furthermore, the age-related suppression of cell-cycle genes in TRAMs was reversed when they were adoptively transferred into a young environment, suggesting a non-cell-autonomous mechanism (Supplemental Figure 3, E and F). In contrast, heterochronic adoptive transfer of TRAMs did not alter the transcriptome of the recipients' AT2 cells (Figure 3, F and $\mathrm{G}$ ). These changes could not be explained by genetic differences between CD45.1 and CD45.2 mice (Supplemental Figure 3, $\mathrm{G}$ and $\mathrm{H}$, and Supplemental Table 4).

Age-related changes in alveolar macrophages are independent of circulating growth factors or cells. Heterochronic parabiosis has long been recognized to reverse and induce age-related phenotypes in old and young adult mice, respectively (23). Because heterochronic parabiosis creates a shared circulation between age-mismatched partners, it provides an approach to determine whether changes in the alveolar microenvironment that shape the alveolar macrophage transcriptome with advanced age are driven by factors in the alveolus or soluble factors or cells from the circulation. Accordingly, we generated heterochronic and isochronic parabiont pairs with stable chimerism in the peripheral blood and flow-sorted TRAMs and AT2 cells 60 days later (Figure 4, A and B). In both TRAMs and AT2 cells, principal component analysis (PCA) and k-means clustering of differentially expressed genes (FDR $q<0.05$ ) showed clustering of the samples according to the age of the host, irrespective of the age of the parabiont pair (Figure 4, A-D). Comparison of TRAM transcriptomes in young/young with old/old parabiont pairs revealed 866 differentially expressed genes (FDR $<0.05$, Figure $4 \mathrm{E}$ ), 474 of which were also identified as differentially expressed in our independent aging data set $(P<$ 0.01, Supplemental Figure 4A). In stark contrast, we found no differentially expressed genes between TRAMs from the old members of the old/old pair and the old members of the young/old pairs (Figure 4F), and only 5 genes were differentially expressed between young members of the young/young pairs versus young members of the young/old parabiont pairs (Figure 4G). The distribution of differentially expressed genes in TRAMs and AT 2 cells between the age-mismatched parabionts and the age-matched parabionts was similar (Supplemental Figure 4C). Heterochronic parabiosis did not reverse the suppression of a curated list of cell-cycle genes in TRAMs (Supplemental Figure 4, D and E). We observed similar results in AT2 cells (Figure 4, H-M, and Supplemental Figure 4B). Differences between CD45.1 and CD45.2 strains did not contribute to the findings (Supplemental Figure $4 \mathrm{~F})$. These findings largely exclude a contribution from circulating factors or cells to the changes in the alveolar macrophage or AT2 transcriptome with advancing age.

The aging microenvironment confers resistance to GM-CSF signaling in alveolar macrophages. Lung macrophage numbers are controlled by GM-CSF; lung epithelium-specific overexpression of the gene encoding GM-CSF (Csf2) in transgenic mice is sufficient to increase alveolar macrophage numbers (24), and genetic loss of Csf2 results in the loss of alveolar macrophages in mice and humans $(13,14)$. GM-CSF also plays an important role in the host response to influenza A infection; transgenic expression of $C s f 2$ in mice accelerates viral clearance and attenuates lung injury after influenza A infection, and genetic loss of Csf2 results in more severe lung injury (25-27). In a murine model of influenza A pneumonia, we reported that, although viral clearance was similar in young adult and aged mice, older mice showed enhanced mortality and worsened lung injury when compared with young adult mice (28). We found that GM-CSF administered intratracheally to mice 1 day before influenza $\mathrm{A}$ infection reduced mortality in both young and aged mice (Figure 5, A and B, and Supplemental Figure 5, A and B).

We sought to determine whether components of the GM-CSF signaling axis were altered as a function of age. The expression of genes known to regulate signaling through GM-CSF or M-CSF (Csf2ra, Csf2rb, Csf1r, Il3ra, and Jak2) was not altered by aging (Figure 5C). Similarly, the expression of Csf2, Csf1, and Il34, genes encoding known ligands of the GM-CSF receptor and M-CSF receptor in AT2 cells, was unchanged during aging (Il3 was not detected) (Figure 5D). We treated mice with exogenous GM-CSF intratracheally and harvested alveolar macrophages 14 days later for RNA-Seq (Figure 5E). Treatment with GM-CSF resulted in changes in the expression of cell-cycle genes in young adult mice, generally favoring a more proliferative phenotype, but these 
A

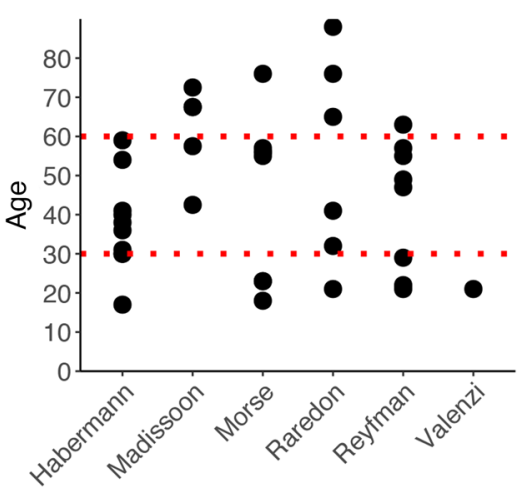

B

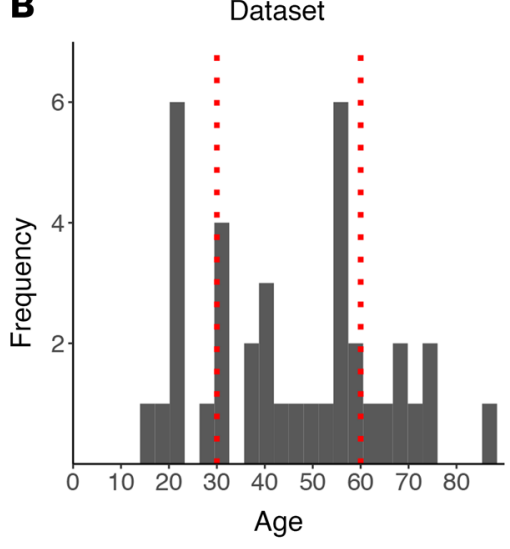

c
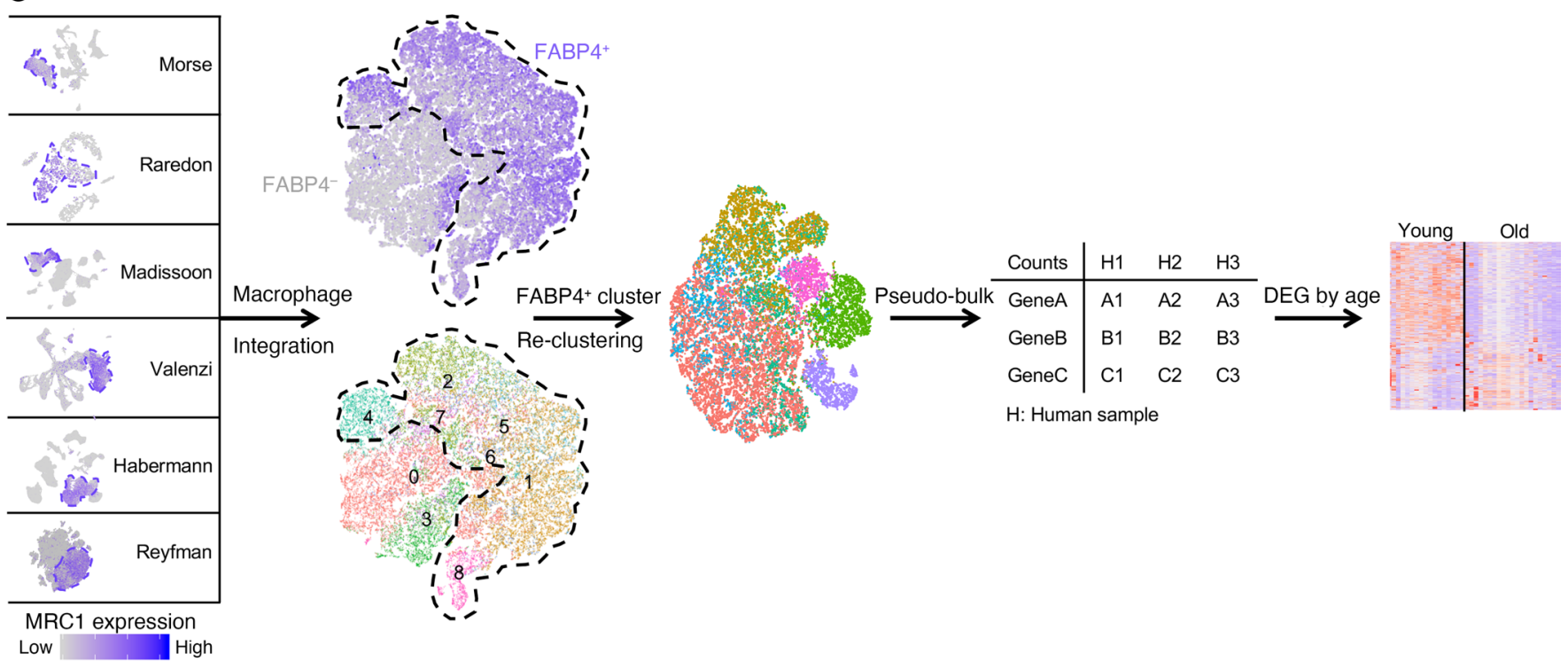

D

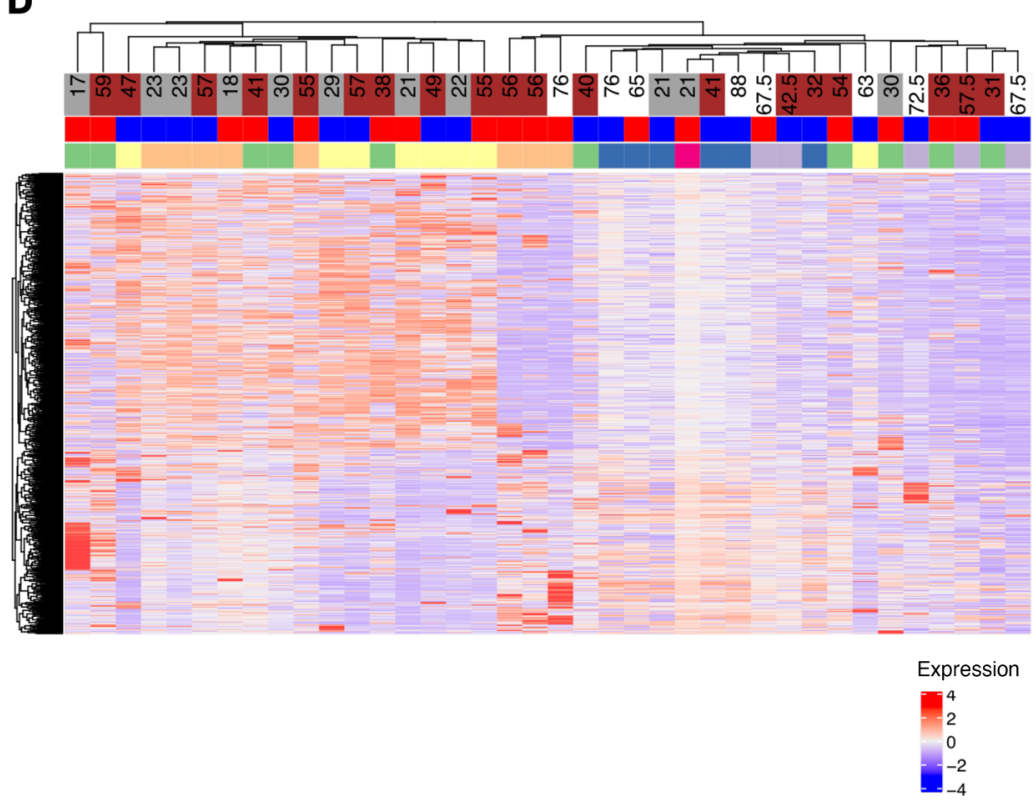

\begin{tabular}{|c|c|c|}
\hline Age (yr) & Sex & Study \\
\hline $\begin{array}{l}O \quad 60+ \\
O 30-60 \\
O \leq 30\end{array}$ & $\begin{array}{l}\text { OFemale } \\
\text { OMale }\end{array}$ & $\begin{array}{l}\text { OHabermann } \\
\text { OMadissoon } \\
\text { OMorse }\end{array}$ \\
\hline
\end{tabular}

Down genes in aging IFG1, PDGFA, C3, EGFR RAB27B, CD36, RAB25, P2RY1, SOD3, CCNT1, CSF1, SIGLEC1, RAB12, HSF4

Up genes in aging IFITM2, TNFAIP8, CTSD, CTSS, ATF3, GPX3 DNAJB4, DNAJB4, HSPB1, HSPA1B, DNAJC10, MARCO

\section{Down GOs in aging}

GO:0045944 positive regulation of transcription by RNA polymerase II GO:0030155 regulation of cell adhesion GO:0042127 regulation of cell

$$
\text { proliferation }
$$

GO:0010628 positive regulation of gene expression

Up GOs in aging

GO:0002252 immune effector process GO:0032680 regulation of tumor necrosis factor production GO:0001775 cell activation 
Figure 2. Integrated analysis of single-cell RNA-Seq data obtained from the healthy human lung reveals uniform changes in the transcriptome of alveolar macrophages with age. (A) Age distribution in each of the 6 published data sets of single-cell RNA-Seq data obtained from healthy human lungs. (B) Histogram of the age distribution in the combined data from the 6 studies. (C) Schematic of the integrated analysis. Alveolar macrophages were identified by expression of typical macrophage marker genes including FABP4 within each of the individual data sets. After reclustering, clusters composed of contaminating cells identified by reduced expression of FABP4 were eliminated. The resulting integrated analysis showed no clustering of alveolar macrophages as a function of age (see also Supplemental Figure 2). Accordingly, cells from each individual were combined to generate a pseudo-bulk transcriptome, and differentially expressed genes with aging were compared. Full code is available on GitHub (https:// github.com/NUPulmonary/Doublehit_Human_scRNA_Analysis; branch - master; commit ID: e486203eb0e1437d73be589a31c803fbc46182bd). (D) Pseudo-bulk analysis of alveolar macrophages from each of the 38 subjects. A heatmap of the differentially expressed genes between individuals under 30 and over 60 years of age was generated. The top columns indicate the chronological age and sex of each subject and the study in which they were included. Down, downregulated; Up, upregulated.

changes were absent in old mice (Figure 5, F-H, and Supplemental Figure 5, C and D).

Changes in composition of the alveolar lining fluid affect alveolar macrophage responses to GM-CSF in aging. Alveolar macrophages reside in the epithelial lining fluid on the apical surface of AT1 and AT2 cells separated from endothelial and mesenchymal cells by highly impermeable tight junctions between these cells (29). We therefore reasoned that changes in the expression of ligandreceptor pairs between alveolar macrophages and AT 2 cells would provide insights into possible mechanisms for the reduced proliferation of alveolar macrophages during aging. We queried a database of known ligand-receptor pairs, in which we identified 72 genes that were changed in AT2 cells with aging. These genes formed 255 receptor-ligand pairings with genes expressed in TRAMs (Figure 6A and refs. 30, 31). A significant proportion of these genes ( 31 of 72 ) encoded proteins in the extracellular matrix or secreted proteins that modify the extracellular matrix (Supplemental Table 15). Differentially expressed genes also included some growth factors (Areg, Ntf3, Vegfa, Igf1) and genes associated with spatial organization (semaphorins and Slit). We then queried a proteomic database of bronchoalveolar lavage (BAL) fluid from mice to identify proteins in our interactome analysis detected in normal BAL fluid (32). These included many of the matrix genes observed in our interactome analysis (Col4a2, Hspg2, B2m, Fga, Fgg, Tgm2, Col3a1, Col6a3, Fbln1, Fn1, Tnc). Furthermore, the levels of these and other matrix proteins were among the most differentially altered in the whole lung proteome of aged compared with young mice (15). We compared the proliferation of BMDMs in response to GM-CSF on plastic, laminin, or collagen matrices or a matrix laid down by a murine AT2-like cell line (MLE-12). We found that proliferation was enhanced when cells were grown on these matrices compared with plastic (Figure 6C).

As excessive matrix would enhance proliferation in aging, we wondered whether other factors in the alveolar lining fluid might inhibit it. Hyaluronan is a ligand of CD44, which is expressed by alveolar macrophages. Hyaluronan is present in the alveolar epithelial lining fluid and has been shown to modulate the behavior of alveolar macrophages during influenza A infection (33). Furthermore, CD44-deficient mice have reduced numbers of alveolar macrophages, suggesting a role for CD44 in alveolar macrophage proliferation (34). We found that the levels of hyaluronan were consistently and significantly increased in aged compared with young adult animals (Figure 6B). The increased proliferation of BMDMs grown on collagen was lost in the presence of hyaluronan, offering one potential explanation for the reduced numbers and proliferation of alveolar macrophages we observed with aging (Figure 6C).

Do changes in alveolar macrophage ontogeny contribute to the loss of alveolar macrophage numbers and function during aging? Sakai et al. reported that monocyte-derived Kupffer cells were transcriptionally different from those populating the liver during development and responded differently to injury (11). An analogous change in the cellular ontogeny of alveolar macrophages the replacement of embryonically derived TRAMs with MoAMs in response to repeated injuries might offer a complementary, cell-autonomous mechanism for changes in alveolar macrophages during aging.

We first asked whether TRAMs are replaced by MoAMs over the course of the lifespan of mice maintained in barrier conditions. We used a genetic lineage-tracing system that combined radiation with thoracic shielding and low-dose systemic busulfan to generate 4-month-old chimeric mice (CD45.1/CD45.2) with complete chimerism in monocytes and approximately $80 \%$ preservation of TRAMs (Supplemental Figure 6A). If TRAMs are slowly replaced by recruited MoAMs over the lifespan, the proportion of MoAMs would increase. In contrast, if TRAMs are more capable of selfrenewal than MoAMs, the small population of MoAMs recruited during the generation of chimeras would decline. However, both cell populations were stable over the 24-month lifespan (Supplemental Figure 6B). Furthermore, 4 months after the generation of bone marrow chimeras ( 6 months of age), MoAMs showed levels of EdU incorporation comparable to levels in TRAMs (Supplemental Figure 6C), confirming the findings of a previous report (35). These findings also argue against enhanced apoptosis of TRAMs as a cause of their reduced numbers during aging, as a loss of alveolar macrophages due to apoptosis leads to the recruitment of monocyte-derived cells (Supplemental Figure 3F).

Laboratory mice are housed in facilities where unusual steps are taken to limit exposure to inhaled pathogens and environmental particulates. Accordingly, we exposed mice to concentrated urban particulate matter air pollution of less than $2.5 \mu \mathrm{m}$ in diameter $\left(\mathrm{PM}_{2.5}\right)$ at a dose, as we previously reported, that induces the release of IL- 6 from alveolar macrophages and enhances the susceptibility to thrombosis ( 10-fold the concentration observed outside our laboratory in Chicago) for 6 hours per day on 3 consecutive days $(36,37)$. As a positive control, we used treatment with intratracheal LPS, which has been shown to induce the recruitment of MoAMs (38). We found that exposure to this level of airborne particles, which is typically encountered in the developing world, did not induce recruitment of MoAMs to the lung or deplete TRAMs (Supplemental Figure 6, D-G). In contrast, the induction of severe lung injury by infection with a sublethal dose of influenza A virus or exposure to a dose of bleomycin that results in transient fibrosis resulted in the recruitment of MoAMs that persisted 
A

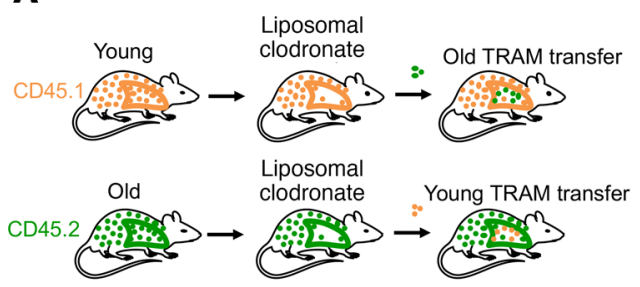

B

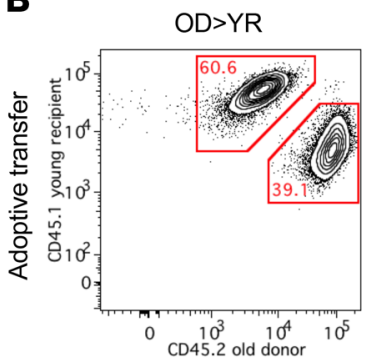

$\mathrm{YD}>\mathrm{OR}$

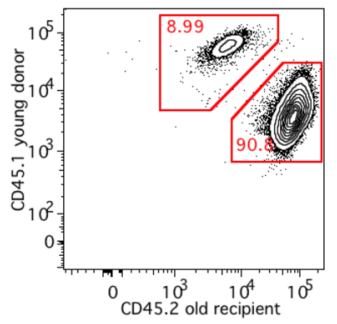

C

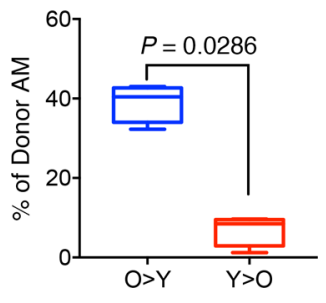

D

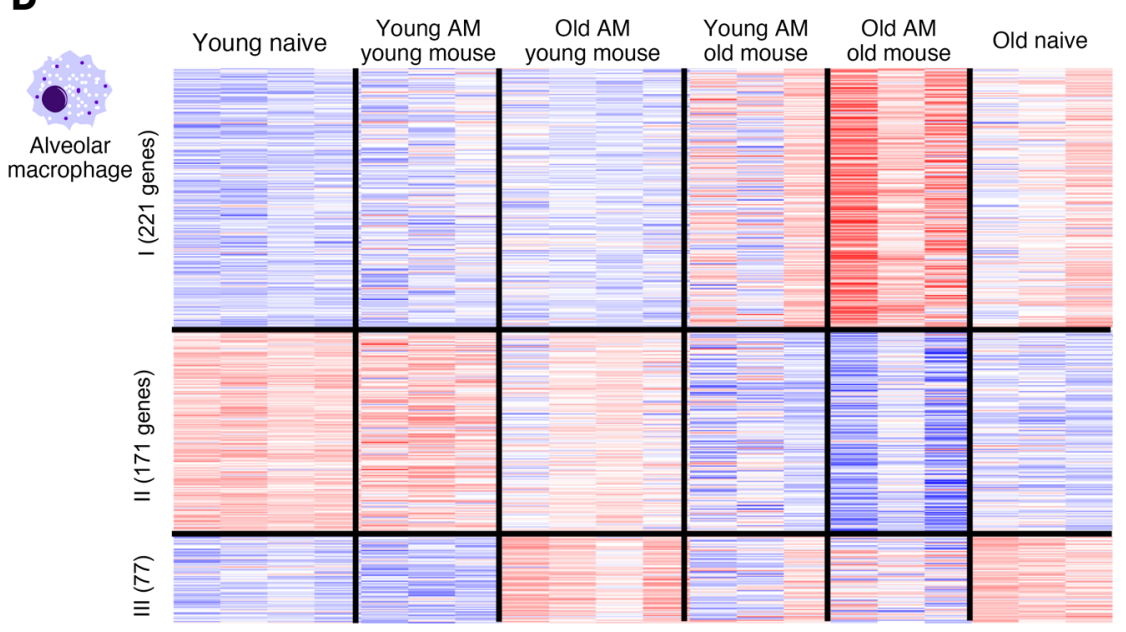

E
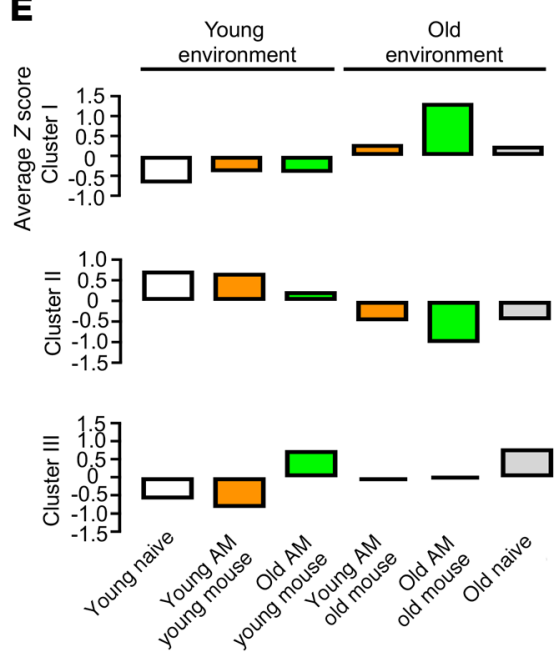

$\mathbf{F}$

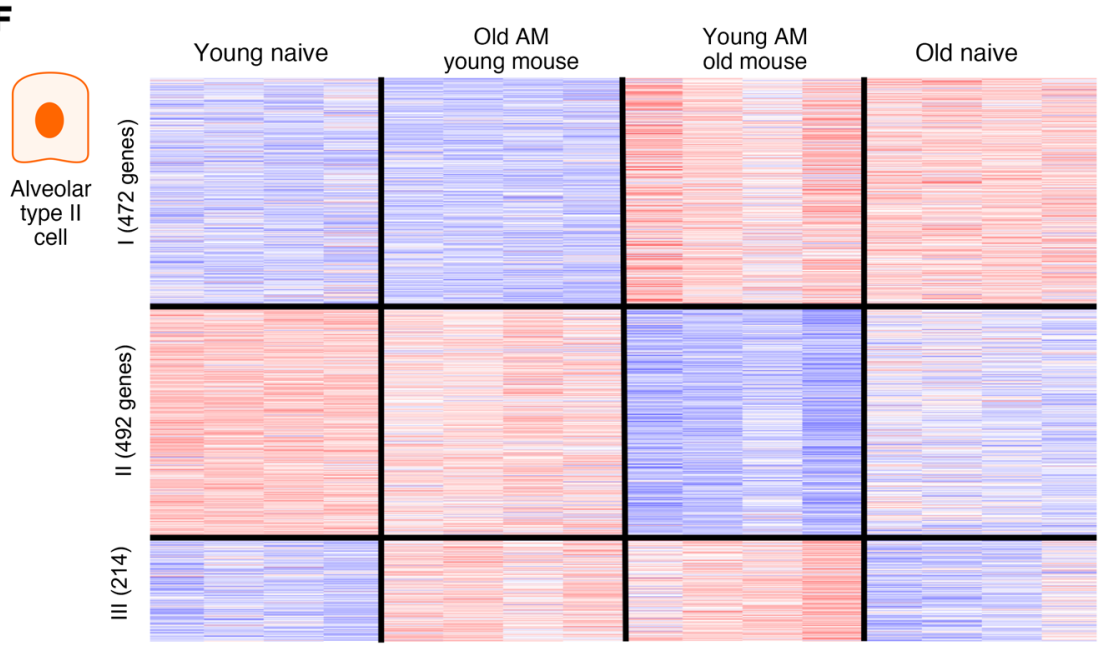

G

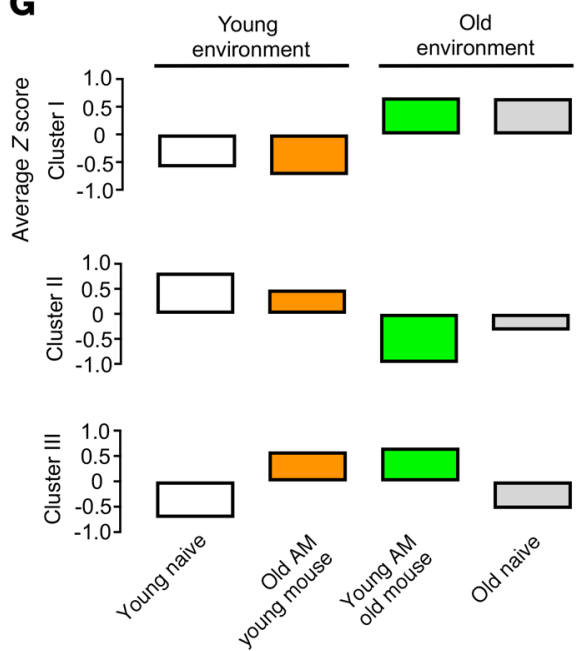

Figure 3. Age-related transcriptomic changes in TRAMs are not cell autonomous. (A) Heterochronic adoptive transfer experiments were performed using CD45.1/CD45.2 pairings as indicated (see also Supplemental Figure 3). (B) Representative flow cytometric plots show engraftment of TRAMs from old (1824 months) (OD) and young adult (4-6 months) (YD) donors into young adult (YR) and old recipients (OR), respectively. Harvesting was performed 60 days after the adoptive transfer. All mice received liposomal clodronate $(25 \mathrm{~L}$ ) intratracheally 72 hours prior to the adoptive transfer (also see Supplemental Figure 3). $n=4$ mice per group. (C) Percentage of engraftment of donor alveolar macrophages (AM) 72 hours after intratracheal adoptive transfer of TRAMs from old donors into young adult recipients (OD>YR) or young adult donors into old recipients (YD>OR). $n=4$ mice per group. Mann-Whitney $U$ test. (D) Heatmap shows k-means clustering of differentially expressed genes (FDR $q<0.05$ in ANOVA-like test) in TRAMs 60 days after heterochronic adoptive transfer into young or old mice. Naive mice did not undergo adoptive transfer. Young and old alveolar macrophages in the same mouse were distinguished by the CD45.1/CD45.2 label (see the full list of genes in Supplemental Table 3). (E) Average z scores for the genes in clusters I, II, and III in D. (F) Heatmap shows k-means clustering of differentially expressed genes (FDR $q<0.05$ in ANOVA-list test) in AT2 cells 60 days after heterochronic adoptive transfer of TRAMs (see the full list of genes in Supplemental Table 3). (C) Average $z$ scores for the genes in clusters I, II, and III from F. 
in the lungs 60 days after the injury (Supplemental Figure 6, $\mathrm{H}$ and I, and ref. 8). These results confirm that alveolar macrophage populations are remarkably stable over the life course outside of a severe lung injury.

We took advantage of the small but stable chimerism in shielded bone marrow chimeric mice to compare transcriptomic changes with aging in MoAMs and TRAMs. Although age-related changes in MoAMs and TRAMs were similar (Figure 7A), a number of genes were differentially expressed between them (clusters IV and V; Supplemental Table 16). These MoAMs were evenly distributed in the lung tissue (Figure 7B), thus, the observed differences likely reflect their ontogeny, independent of the microenvironment. Consistent with this hypothesis, we observed significant overlap between the genes in clusters IV and V with genes we previously reported to be differentially expressed between TRAMs and MoAMs 10 months after bleomycin-induced lung injury (Figure 7C and ref. 8), and we reidentified all of the genes that differed between MoAMs and TRAMs after the intratracheal administration of liposomal clodronate in a previous study using microarray (39). These changes were not explained by differences between the CD45.1 and CD45.2 strains (Supplemental Figure 7). To determine whether these changes were driven by changes in DNA methylation, we performed reduced representation bisulfite sequencing, which provides a genome-wide assessment of DNA methylation, on TRAMs and MoAMs from aged ( 18 months old) chimeric animals. We looked for evidence of differential DNA methylation in promoter regions $1000 \mathrm{bp}$ upstream and downstream of the start site of differentially expressed genes in our RNA-Seq data set, and in putative enhancer regions specific to alveolar macrophages defined as H3K4me1 peaks identified by Lavin et al. $(7,40)$. We found no evidence of differences in these regions (Figure 7D).

Ontogeny does not impact the response of alveolar macrophages to influenza A viral infection or bleomycin-induced fibrosis. We wanted to test whether ontological differences between TRAMs and MoAMs alters their response to a subsequent environmental challenge. We generated a cohort of shielded chimeric mice and subjected them to sequential injury with either influenza A infection followed 60 days later by bleomycin, or 2 sequential doses of intratracheal bleomycin separated by a 60-day interval (Figure $8, \mathrm{~A}-\mathrm{C})$. We analyzed TRAMs and MoAMs in these models using RNA-Seq 4 days after influenza A infection and 21 days after bleomycin, reflecting the time when there is maximal lung injury and fibrosis, respectively (8). PCA of the entire data set demonstrated excellent reproducibility in the data (Figure 8, D and E). Consistent with reports from our group and others, newly recruited MoAMs exhibited distinct transcriptional responses characterized by increased expression of inflammatory and fibrotic genes, respectively, during acute injury (Supplemental Figure 8, A and B, and refs. 8,41 ).

We then compared the response of TRAMs and "newly tissue-resident" MoAMs (mTRAMs) (recruited after the first injury) with a subsequent challenge with bleomycin. Prior to the second challenge, we confirmed that MoAMs persisted in the lungs, where they were localized to sites of injury (Supplemental Figure 8C). At this time, there were fewer differentially expressed genes between MoAMs and TRAMs than we observed early after the first injury, but they both still differed when compared with TRAMs from untreated mice (Supplemental Figure 8, D and E, and Supplemental Tables 21 and 22). The response of these mTRAMs to a second challenge with bleomycin was largely similar to that of TRAMs present before the first challenge, whether they were recruited in response to influenza A infection (Figure $8 \mathrm{~F}$ ) or bleomycin instillation (Figure $8 \mathrm{G}$ ). Although there were differences in gene expression between TRAMs and mTRAMs, these changes largely overlapped, irrespective of the injury, and overlapped with differences we observed between TRAMs and mTRAMs in normal aging (Supplemental Figure 8F). Collectively, these results reveal that alveolar macrophage responses to lung injury were similar, irrespective of their ontogeny.

The microenvironment shapes the response of MoAMs and TRAMs to repeated injury. We and others have reported that deletion of MoAMs attenuates the severity of both bleomycin-induced lung fibrosis and influenza A virus-induced pneumonia $(8,26$, 38). Independently, DeGryse et. al. found that repeated administration of bleomycin after recovery results in more severe and persistent injury when compared with a single dose of bleomycin (42). Accordingly, we compared MoAMs recruited to the lungs in response to a single bleomycin challenge with those recruited after a second bleomycin challenge (Figure 9A). MoAMs recruited after a second challenge expressed higher levels of genes and GO biological processes associated with fibrosis (Figure 9, B and C). This response was specific to historic bleomycin, however, as the response of MoAMs to a secondary challenge with bleomycin after historic influenza $A$ infection was almost identical to that seen in naive mice (Figure 9D). Consistent with this finding, the severity of lung fibrosis as measured by lung compliance and soluble collagen was worse 14 days after bleomycin administration in mice that had received historic bleomycin compared with those who did not (Figure 9, E and F), but this was not observed after historic influenza A infection (Supplemental Figure 9, A and B). In contrast, TRAMs and mTRAMs showed evidence of reduced inflammatory gene expression after the second challenge with bleomycin compared with the first challenge, whether the first challenge was influenza A virus infection or bleomycin exposure (Figure 9, G-I, and Supplemental Figure 9, B-E). These results further highlight the importance of the alveolar microenvironment in shaping innate immune memory and innate immune tolerance after repeated inhaled environmental challenges.

\section{Discussion}

As the COVID-19 pandemic unfolds, pneumonia has become a leading cause of worldwide death and will likely remain so for some time. Like other viral and bacterial pathogens, the morbidity and mortality attributable to severe acute respiratory syndrome coronavirus 2-induced (SARS-CoV-2-induced) pneumonia disproportionately affect the elderly $(1,43,44)$. Because alveolar macrophages play a critical role in the response to pathogenic viruses and bacteria that cause pneumonia (3), including SARS$\mathrm{CoV}-2$, reductions in alveolar macrophage numbers or function during aging might underlie some of the enhanced susceptibility of the elderly to pneumonia.

We found that the number of TRAMs and their expression of cell-cycle genes was reduced in old compared with young adult 
A

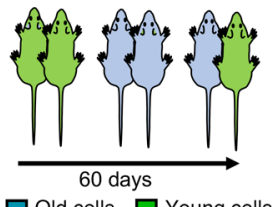

$\square$ Old cells $\square$ Young cells

B

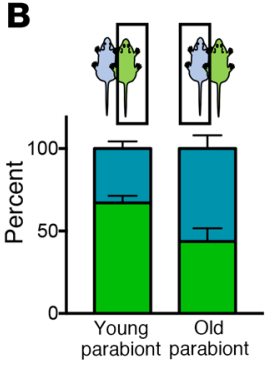

E

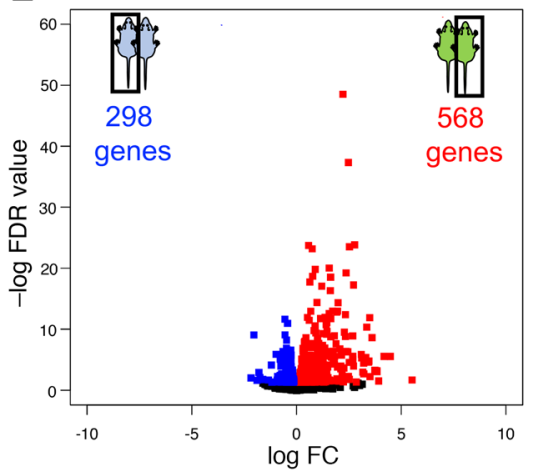

H

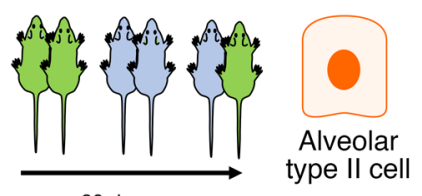

C

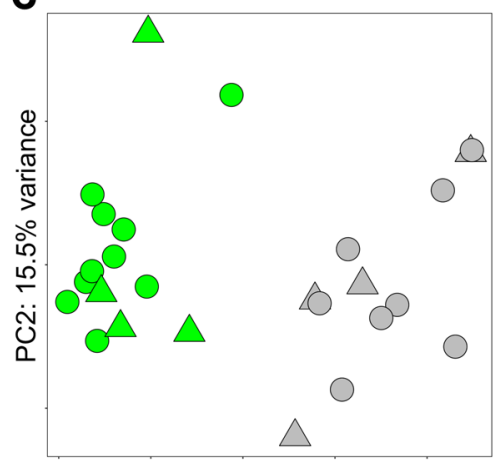

PC1: 24.1\% variance

$\triangle$ Pair $\quad$ Age

Olsochronic Old

$\mathbf{F}$

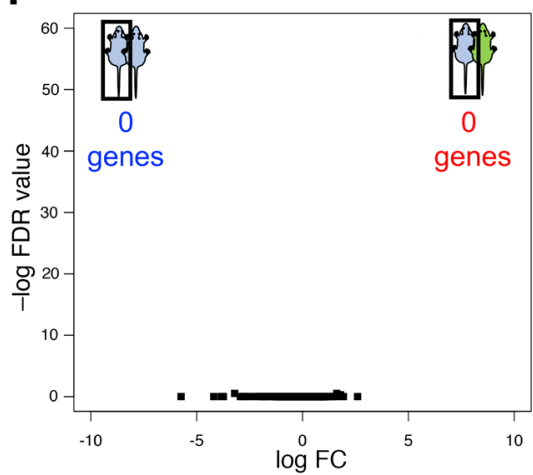

D

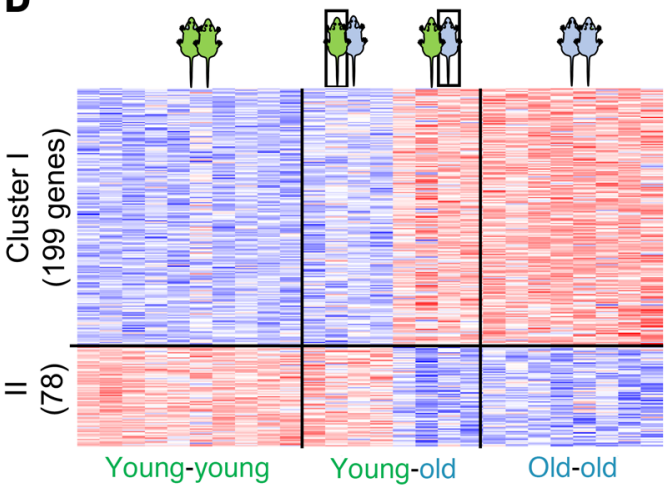

G

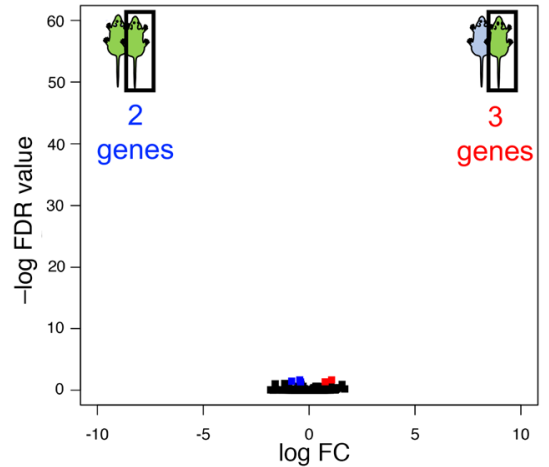

I

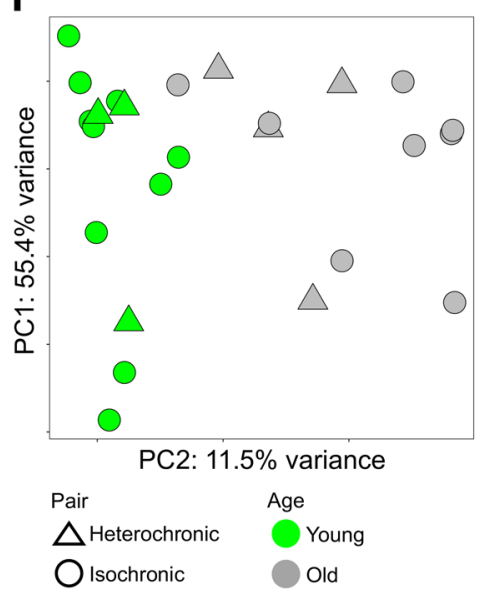

J

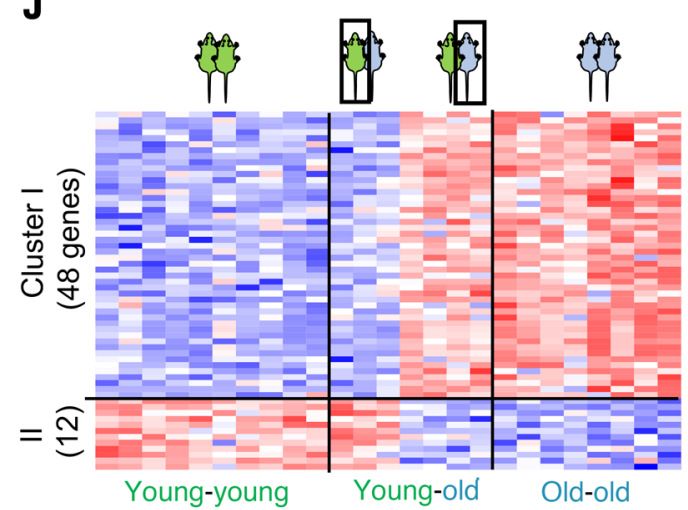

$\mathbf{L}$

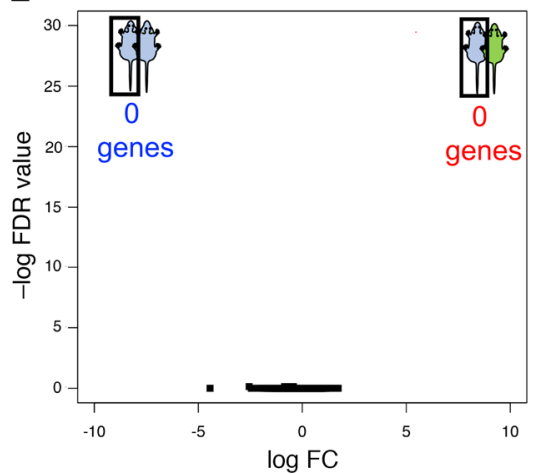

M

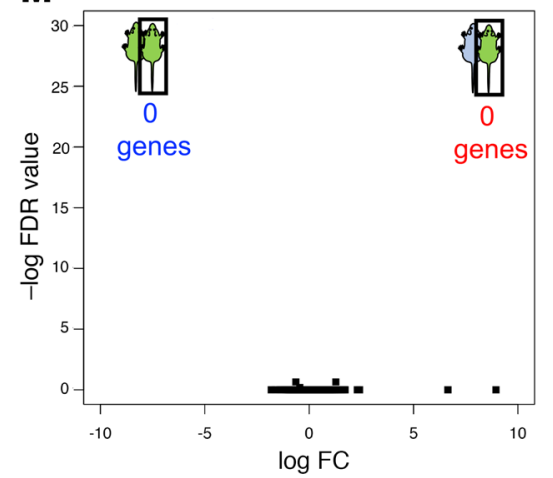


Figure 4. Heterochronic parabiosis does not reverse age-related transcriptomic changes in TRAMs or AT2 cells. (A) Parabionts were generated from young adult (4-6 months, green) and old (18-24 months, gray) pairs, and TRAMs and AT2 cells were harvested after 60 days. (B) Percentage of circulating $\mathrm{CD} 45^{+}$cells from the young or old parabiont pair determined by flow cytometry using CD45.1/CD45.2. $P=$ NS by ANOVA. (C) PCA plot (PC1 and PC2) of TRAM transcriptomes of young and old mice linked to an isochronic or heterochronic parabiont pair. Each symbol represents an individual animal. (D) Heatmap shows k-means clustering of differentially expressed genes in TRAMs (FDR $<0.01$ in ANOVA-like test) between old and young mice with isochronic or heterochronic parabiont pairs (see also Supplemental Table 5). (E-G) Volcano plots show differentially expressed genes in TRAMs from young-young versus old/old versus young/old parabiotic pairs (FDR < 0.05) (see also Supplemental Figure 4 and Supplemental Tables 6-8). (H) AT2 cells were harvested from the same parabiont pairs as in $\mathbf{A}$ after 60 days. (I) PCA plot of transcriptomes of AT2 cells from young and old mice linked to an isochronic or heterochronic parabiont pair. Each symbol represents an individual animal. (J) Heatmap shows k-means clustering of differentially expressed genes in AT2 cells (FDR $<0.01$ in ANOVA-like test) between old and young mice with isochronic or heterochronic parabiont pairs (see also Supplemental Table 9). (K-M) Volcano plot showing differentially expressed genes in AT2 cells from young/young versus old/old versus young/old parabiotic pairs $(F D R<0.05)$ (see also Supplemental Figure 4 and Supplemental Tables 10-12).

mice. These changes were not cell autonomous, as the reduced expression of cell-cycle genes was reversed when alveolar macrophages from young adult mice were adoptively transferred into the lungs of old mice and vice versa. Further supporting a loss of growth or survival signals for alveolar macrophages with advancing age, the engraftment of adoptively transferred alveolar macrophages was much less efficient in old mice than in young adult mice. Heterochronic parabiosis, in which circulating factors and cells were shared between young adult and old animals, had remarkably little effect on the age-related changes in the transcriptome of either alveolar macrophages or AT2 cells.

Alveolar macrophages reside in or near an air-liquid interface formed by the epithelial lining fluid and interact directly with AT1 and AT2 epithelial cells that line the surface of the alveolus, the latter serving as the main source of GM-CSF in the healthy lung. We performed a ligand-receptor analysis of interactions between AT 2 cells and alveolar macrophages with advancing age and found a striking number of ligand-receptor pairs involving genes associated with the extracellular matrix. Consistent with our findings, a published proteomic analysis of the aging mouse lung identified extracellular matrix proteins as among the most differentially abundant during aging (32). Ingber and colleagues discovered that integrin binding to extracellular matrix proteins dramatically changes the response to growth factor signaling, a finding generalized to almost all epithelia (45). However, with the exception of osteoclasts, which are tissue-resident macrophages in the bone, the importance of matrix interactions in the proliferation of macrophages has not been previously reported (46). We found that BMDMs cultured in the presence of GM-CSF showed enhanced proliferation on matrices composed of laminin or collagen when compared with plastic. This enhanced proliferation was inhibited by hyaluronan, which interacts with CD44 that is abundantly expressed in alveolar macrophages (33). We found that hyaluronan was increased several-fold in BAL fluid from old versus young adult mice. This finding complements those of Bell et al., who recently reported that hyaluronan accumulates after influenza $\mathrm{A}$ infection and that the administration of hyaluronidase after viral clearance in a murine model of influenza $\mathrm{A}$ infection accelerates repair via its effects on alveolar macrophages (33).

Long-lived macrophage populations in the lung, brain, and liver are derived from myeloid progenitors originating from the yolk sac or fetal liver (4-6). We show that alveolar macrophages persisted in the murine lung over the lifespan without input from circulating monocytes, even in animals exposed to short-term, high levels of air pollution. However, we found that severe injury could deplete TRAMs and induce the recruitment of bone marrow-derived alveolar macrophages to the lung. Guided by signals from the local microenvironment, these alveolar macrophages reshape their epigenome and obtain gene expression profiles and surface markers resembling mature TRAMs (7). We found no evidence that these cells retain an epigenetic memory of their origin as inflammatory monocytes. Instead, their response to environmental challenge with influenza A virus or bleomycin was almost indistinguishable from that of embryonically derived TRAMs. These findings further implicate the aging alveolar microenvironment in the changes in alveolar macrophage numbers and function with advancing age.

Previous environmental challenge can prime alveolar macrophages and modulate their response to subsequent challenge, a process referred to as "trained immunity" or "innate immune memory" (47). We found that the lung microenvironment strongly modified the response of both TRAMs and MoAMs to a second environmental challenge, suggesting that innate immune memory in alveolar macrophages is also conferred by the local microenvironment, as has been previously suggested (48). Interestingly, the environment differentially affected TRAMs and newly recruited MoAMs. Specifically, TRAMs exhibited reduced expression of inflammatory and fibrotic genes (immune tolerance), irrespective of the type of historic injury or their developmental ontogeny. In contrast, MoAMs recruited after a secondary exposure to bleomycin showed enhanced fibrotic signatures.

Our study has limitations. First, our integrated analysis of single-cell RNA-Seq data from alveolar macrophages in the healthy human lung is limited by the number of published samples. Second, we could not tell whether the accumulation of hyaluronan was secondary to increased synthesis or impaired clearance, and there are no accepted techniques to manipulate the aging extracellular matrix in vivo. Genetic deletions of different matrix components in aged mice will be necessary to identify factors that drive changes in alveolar macrophage phenotype and function with age. These studies will be informed by proteomics studies of changes in the extracellular matrix and epithelial lining fluid in aging mice and humans. Third, we observed reproducible transcriptomic differences between MoAMs and TRAMs that did not affect the response to bleomycin or influenza A infection and were not associated with detectable changes in DNA methylation at a genome scale. It is possible, however, that other epigenetic differences, such as histone modifications, as has been previously described (7), are present between TRAMs and MoAMs, and some of these might shape the response to other inhaled toxins or 
A

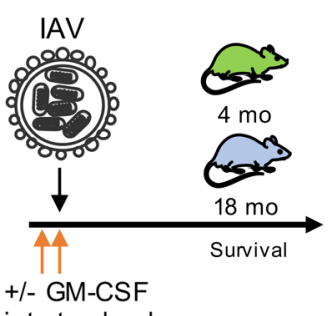

intratracheal
B

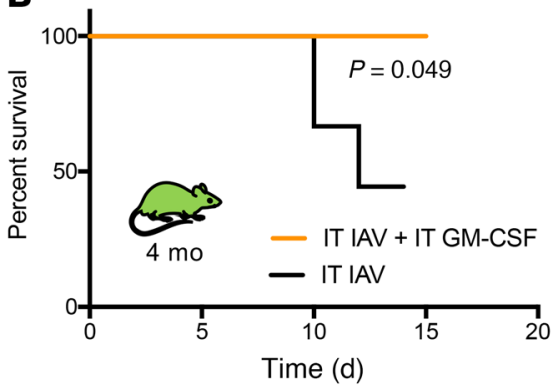

D

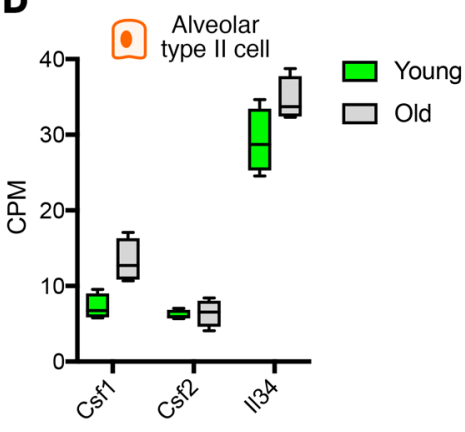

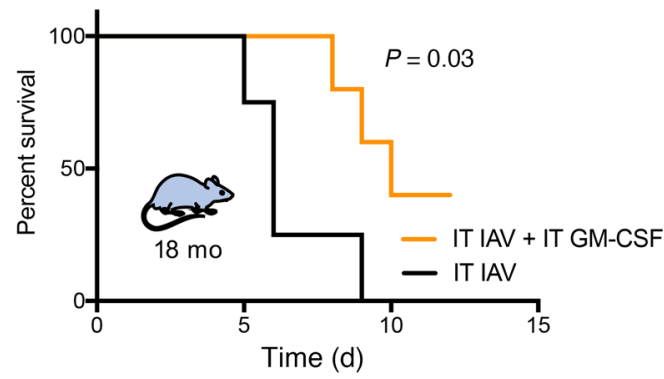

E

GM-CSF

intratracheal

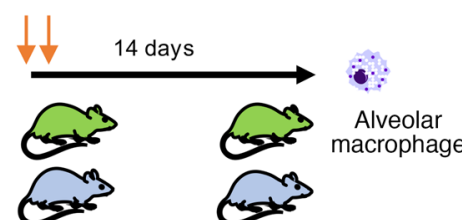

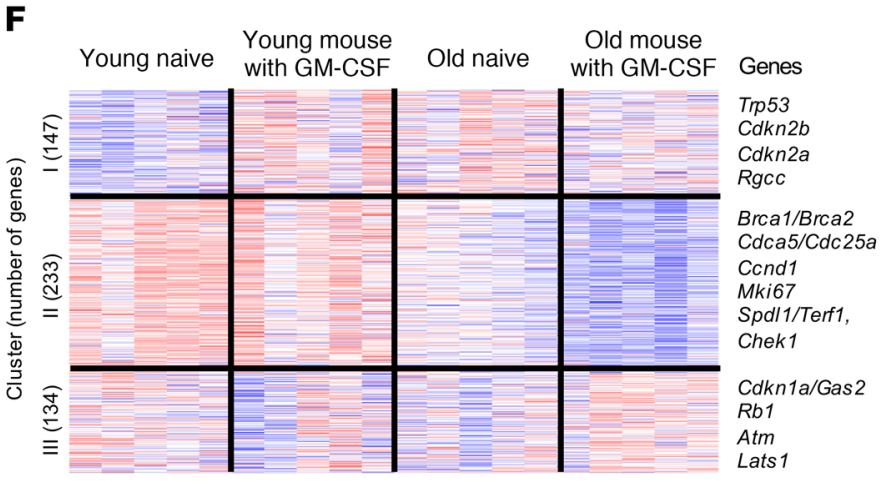

$\mathbf{F}$
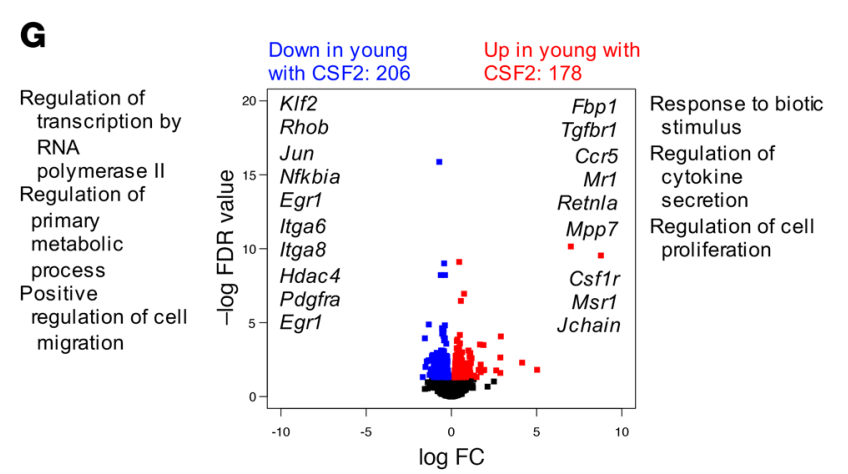

H

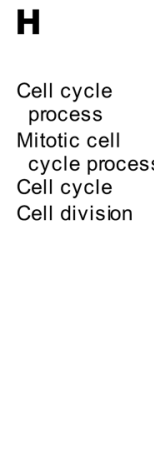

GO processes

Negative regulation of cell cycle

Negative regulation of cell proliferation

Negative regulation of cell cycle phase transition

Positive regulation of cell cycle

Microtubule cytoskeleton organization involved in mitosis

Positive regulation of mitotic cell cycle phase transition

Chromosome segregation

Negative regulation of cell cycle

Cell cycle arrest

Cell cycle checkpoin

Cellular response to radiation

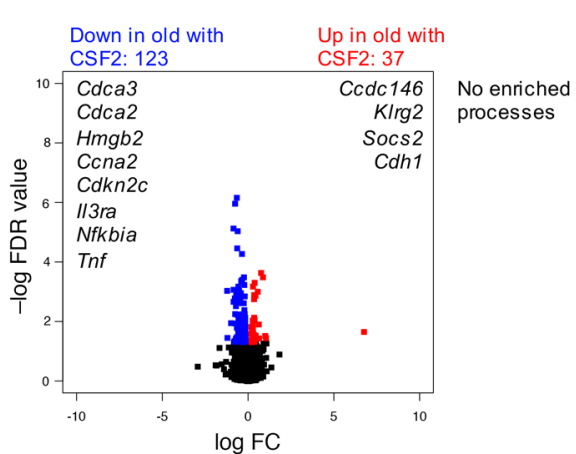

Figure 5. The aging microenvironment confers resistance to GM-CSF signaling in alveolar macrophages. (A) Schematic of the experimental design for B. Green represents young adult (4-6 months) mice, and gray represents old (18-24 months) mice. (B) Survival curve for young adult (4 months) or old (18 months) mice intratracheally infected with influenza A virus (A/WSN/33), $25 \mathrm{PFU} /$ animal, with or without intratracheal CM-CSF (5 mg/kg). $n=5$ per group. Mantel-Cox log-rank test. (C) Box-and-whisker plot shows the expression of genes known to regulate signaling through the GM-CSF receptor (Csf2ra, Csf2rb) and the M-CSF receptor (Csf1r) in TRAMs from young and old naive mice ( $n=3-4$ mice per group). FDR $>0.05$ after multipair $t$ test adjustment. (D) Box-and-whisker plot showing expression of $C s f 1, C s f 2$, and $/ 134$ in AT2 cells from young and old naive mice ( $n=4$ mice per group). FDR $>0.05$ after multipair $t$ test adjustment. (E) Schematic for F-H. Young adult (4 months) and old (18 months) mice were treated with intratracheal GM-CSF (5 mg/kg), and alveolar macrophages were harvested 14 days later (see also Supplemental Figure 5C). (F) Heatmap shows k-means clustering of cell-cycle genes between TRAMs from GM-CSF-treated and untreated young adult and old mice. Representative genes and GO processes are shown (see also Supplemental Table 13). (G) Volcano plot shows differentially expressed genes in young mice (FDR $q<0.05$ ) after treatment with intratracheal CM-CSF (see also Supplemental Table 14). (H) Volcano plot shows differentially expressed genes in old mice (FDR $q<0.05$ ) after treatment with intratracheal GM-CSF (see also Supplemental Table 14). CPM, counts per million reads. 
A

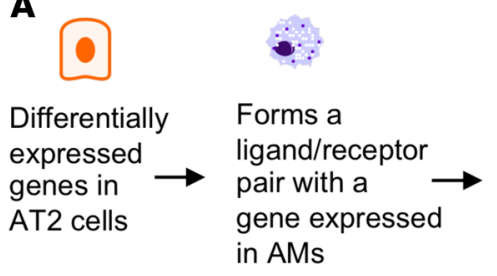

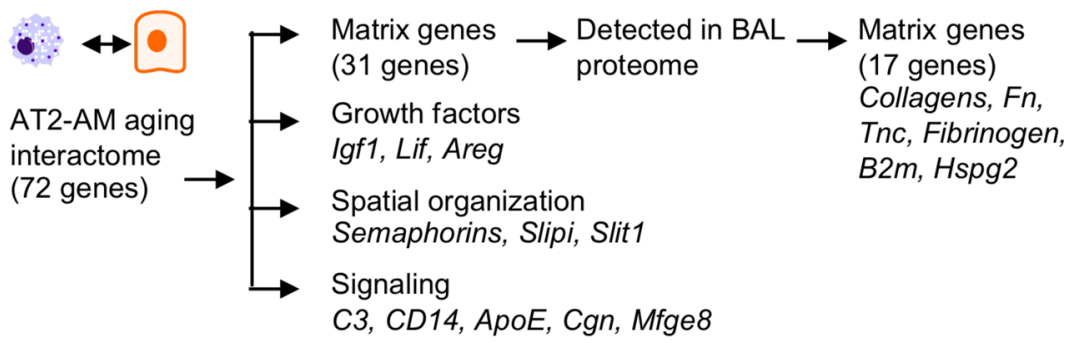

B

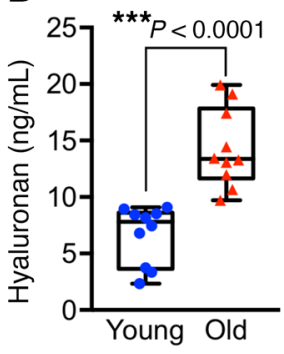

C

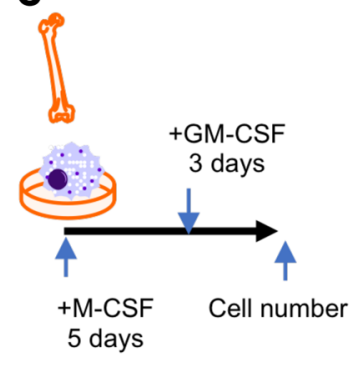

Hyaluronan
Hyaluronidase

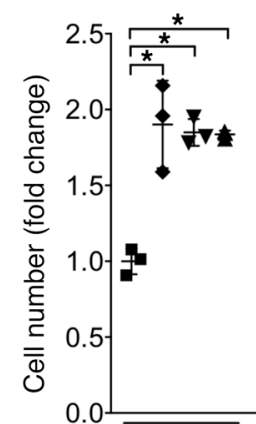

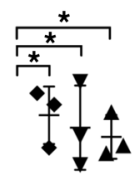

- Plastic

- MLE12 extracellular matrix

$\checkmark$ Laminin

A Collagen type IV

Figure 6. The presence of high-molecular-weight hyaluronan reduces proliferation in BMDMs. (A) Schematic of ligand-receptor analysis of AT2 cells and alveolar macrophages during aging. Analysis of differentially expressed genes (FDR < 0.01 ) in AT2 cells from old (18-24 months) and young adult (4-6 months) mice that were part of a ligand-receptor pair for which the corresponding ligand or receptor was detected in alveolar macrophages identified 72 genes with 255 possible interactions. Thirty-one of these genes encoded matrix proteins, 17 of which were detected in proteomics analysis of BAL fluid from healthy mice, reflecting the composition of the extracellular lining fluid. See Changes in composition of the alveolar lining fluid affect alveolar macrophage responses to CM-CSF in aging in Results and Supplemental Table 20 for details. (B) Levels of total hyaluronan in BAL fluid from young (4-6 months) and old (18-24 months) mice ( $n=10$ mice, 5 male and 5 female per age). ${ }^{* *} P<0.0001$, by Student's $t$ test. (C) BMDMs were grown with M-CSF $(5 \mathrm{ng} / \mathrm{mL})$ for 4 days, replated, and then stimulated with GM-CSF $(5 \mathrm{ng} / \mathrm{mL})$ for 3 days, after which the BMDMs were quantified $(n=6$ replicates per experiment). Bar plots show the fold change in cell numbers when cells were treated with GM-CSF on plates coated with matrix from the mouse lung alveolar epithelium-like cell line MLE-12, laminin, and collagen (both $\left.1 \mu \mathrm{g} / \mathrm{cm}^{2}\right)$ in the presence or absence of high-molecular-weight hyaluronan $\left(1 \mu \mathrm{g} / \mathrm{cm}^{2}\right)$. Averages and the standard error of 3 independent experiments are shown. ${ }^{*} P<0.05$, by 2 -way ANOVA followed by Student's $t$ test.

pathogens. Last, we demonstrated that MoAMs recruited in mice at 8 weeks of age (the age when we induced bone marrow chimerism) persisted over the lifespan (24 months), but the ontogeny of alveolar macrophages may change between development and 2 months of age.

In conclusion, our study shows that, although alveolar macrophages are long-lived lung-resident cells, changes in their number and transcriptional identity with advancing age are not cell autonomous but are instead shaped by the alveolar microenvironment in which they reside, independent of signaling molecules or cells in the circulation. These findings highlight the importance of understanding and targeting aberrant signals from the aging lung, including the extracellular matrix, if we are to mitigate the impact of aging on the risk of severe viral pneumonia.

\section{Methods}

Mice. C57BL/6J (Jax 000664) and CD45.1 (Jax 002014) mice were bred in our facility, and our colonies are refreshed yearly with mice purchased from The Jackson Laboratory. Transgenic mice expressing a nuclear, chromatin-bound GFP reporter (H2B-GFP) driven by the murine surfactant protein $\mathrm{C}$ (SPC) promoter (SPC H2B-GFP) were a gift of Carla Kim (Harvard Medical School, Boston, Massachusetts, USA) (49). When indicated, young and aged C57BL/6 mice from the
NIH's National Institute on Aging (NIA) colony were used. The number of animals per group was determined on the basis of our previous publications. Investigators were not blinded to the group allocation. Mice were housed at the Center for Comparative Medicine at Northwestern University in microisolator cages. Mice were maintained under a standard 12-hour light/12-hour dark cycle and an ambient temperature of $23^{\circ} \mathrm{C}$ and were provided a standard rodent diet (Teklad LM-485, Envigo) and water ad libitum. All mice were male unless otherwise indicated in the figure legends.

Murine model of PM exposure. Inhalational exposure to $\mathrm{PM}_{2.5}$ concentrated ambient particles (CAPs) was performed as previously described (36). Briefly, mice were housed 8 hours per day for 3 consecutive days in a chamber connected to a versatile aerosol concentration and exposure system (VACES). We exposed control mice to filtered air in an identical chamber connected to the VACES, in which a Teflon filter was placed on the inlet valve to remove all particles. We estimated ambient $\mathrm{PM}_{2.5}$ concentrations as the mean of reported values from the 4 Environmental Protection Agency (EPA) monitoring locations closest to our location. The mean concentration in the PM exposure chamber was $118.3 \pm 5.21 \mathrm{mg} / \mathrm{m}^{3}$. Fluorescence labeling of resident alveolar macrophages with PKH26 in vivo was performed as previously described (50). Three treatment groups were evaluated: (a) the control group with intratracheal instillation of $50 \mu \mathrm{L}$ PBS, (b) the 
A

GO processes

Mitochondrial respiratory chain complex I assembly Antigen processing and presentation

Complement activation

Cholesterol metabolic

$$
\text { process }
$$

Positive regulation of transcription by RNA polymerase II

Chromatin organization

Histone modification

Positive regulation of cell motility

Cell migration

Regulation of cell

proliferation

Mitotic cell process Cell division

Lipid catabolic process Ion transport
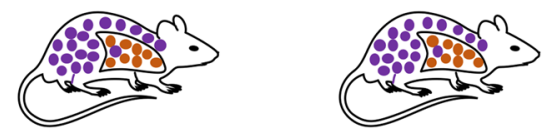

$3 \mathrm{mo}$

$6 \mathrm{mo}$
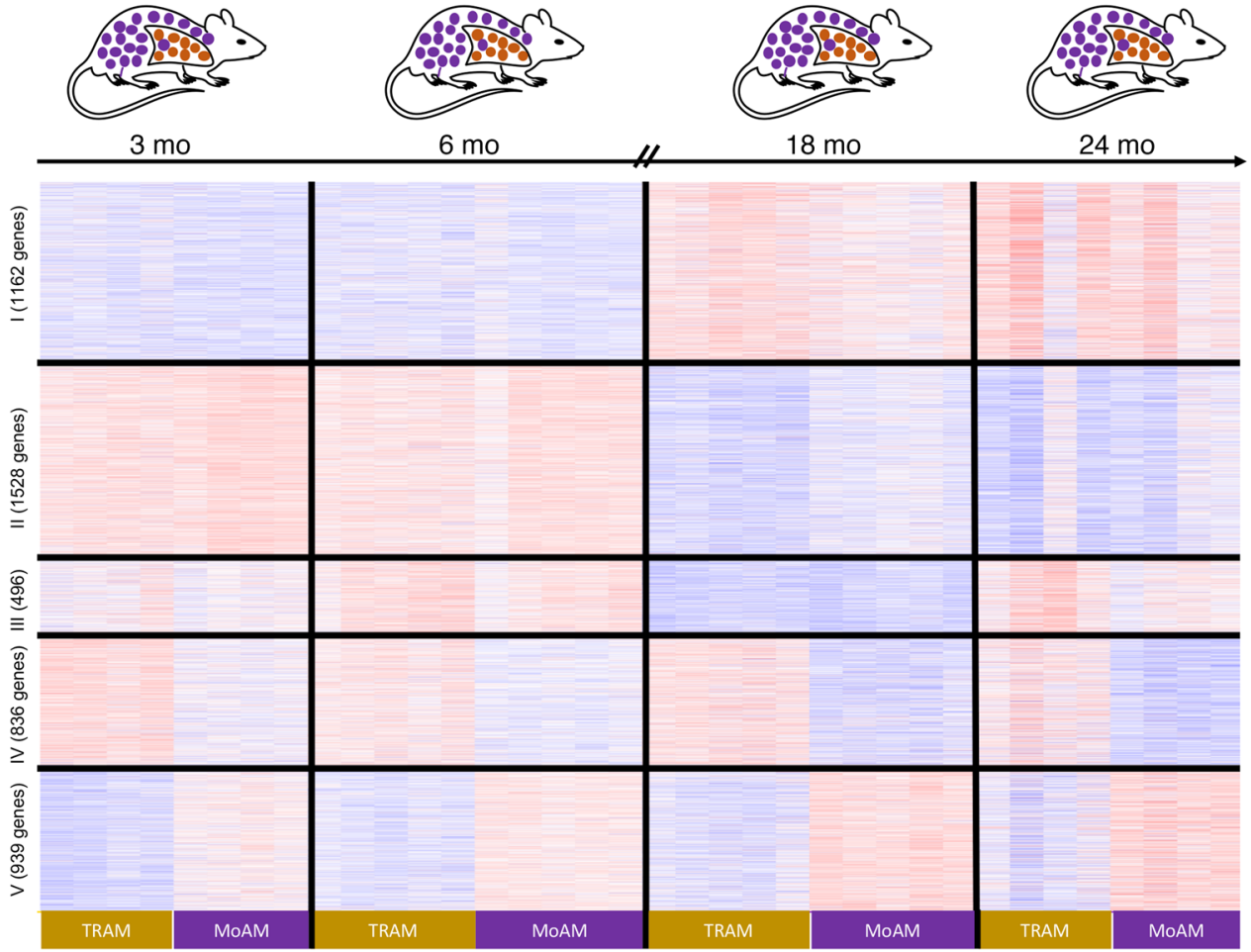

Genes

Nudfa1

Stat3

Sod2/Sod1

Csf1

CD200r1

CD68

Msr1

Sp1

Cdk12

Cdk12

gibrt

Mert

Pten

Rxra

Apoe KIf4

Notch4

Dnmt1

CD163

Csf1

Ccna2

Clec4a2

Cd36

Hmox1

Sirt2

Ripk1

Marco
B

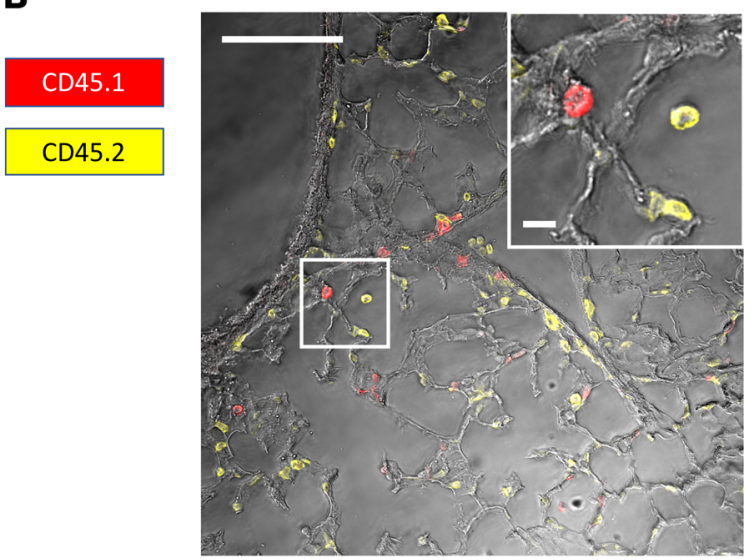

C

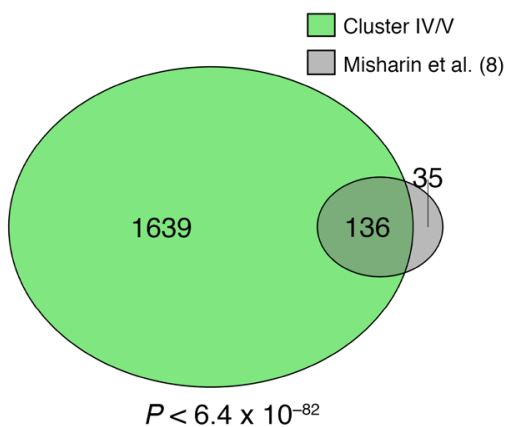

D
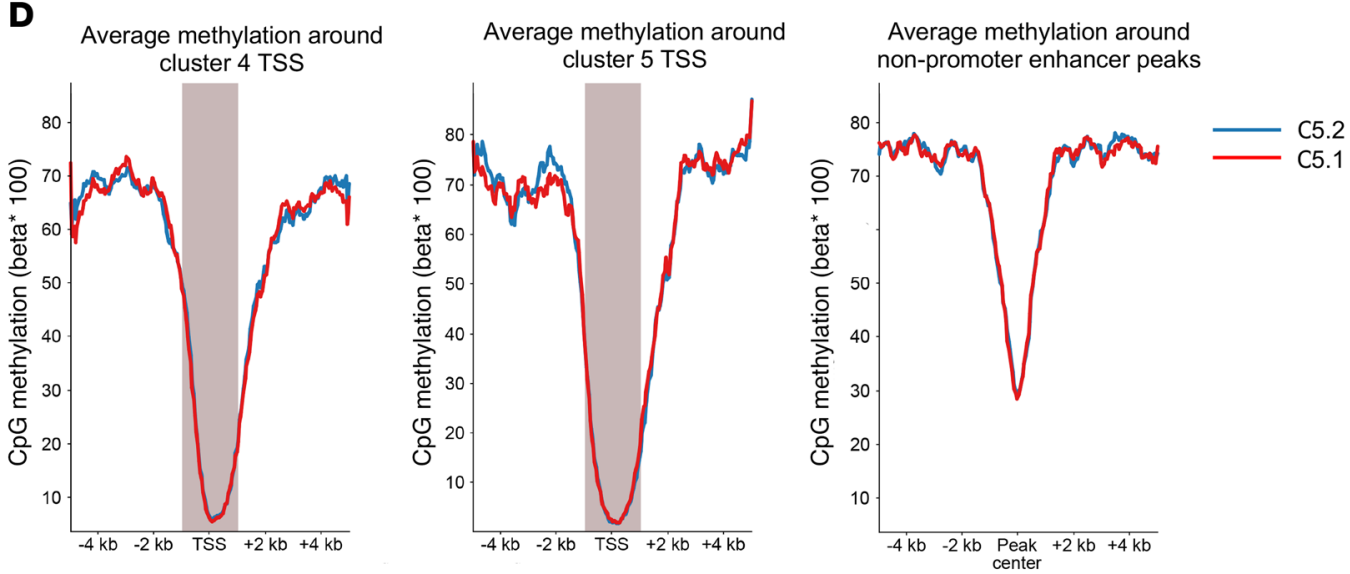
Figure 7. Transcriptional differences between MoAMs and TRAMs persist over the lifespan. (A) Alveolar macrophages from shielded chimeric mice were harvested from mice at the indicated ages, and TRAMs and MoAMs were flow-sorted on the basis of CD45.2 or CD45.1 labeling, respectively. Differentially expressed genes (FDR $<0.01$ in an ANOVA-like test) were identified and subjected to k-means clustering. Selected genes and GO processes from each cluster are highlighted (see Supplemental Table 17 for the full list of genes and GO processes). (B) Representative immunofluorescence image of a lung section from a shielded 6-month-old chimeric mouse. Staining for CD45.2 was done to mark TRAMs and for CD45.1 to mark MoAMs. A combined image overlaid on a phase-contrast image is shown. Scale bars: $40 \mu \mathrm{m}$ and $10 \mu \mathrm{m}$ (enlarged inset). (C) Venn diagram shows overlap of differentially expressed genes between MoAMs and TRAMs in this model (clusters IV and V in A) with an independent data set from Misharin et al. (8) collected 10 months after bleomycin exposure. (D) Reduced representation bisulfite sequencing was performed on TRAMs and MoAMs from 6-month-old mice. The frequency of methylated CpC motifs in promoter regions within 1000 bp upstream and downstream of the transcriptional start site (TSS) of differentially expressed genes between TRAMs and MoAMs in shielded chimeric mice was compared with their frequency across the genome. A similar analysis was performed using putative enhancer regions specific to alveolar macrophages defined as consensus H3K4me1 peaks by Lavin et al. (7). No significant differences in DNA methylation were detected.

LPS treatment group with intratracheal instillation of $1 \mathrm{mg} / \mathrm{kg}$ LPS, and (c) the PM exposure group, with mice placed inside the PM concentrator for 6 hours per day for 3 consecutive days. For the PKH26 experiments, alveolar macrophages were collected by BAL. Cells were then processed and analyzed by flow cytometry (LSR II, Digital FACS instrument, BD) using the following antibodies: APC/Cy7 anti-mouse Ly-6G antibody (BioLegend), Alexa Fluor 647 anti-mouse F4/80 antibody (BioLegend), and Helix NP Green (BioLegend), which is a green-emitting nucleic acid live/dead stain.

$B A L$ and determination of hyaluronan concentration. Eight- to 12-week or 18-month-old naive mice were anesthetized, intubated, and infused with $1 \mathrm{~mL}$ PBS via intratracheal injection as previously described $(36,37)$. The BAL solution was then recovered, and the concentration of hyaluronan with a molecular size over $35 \mathrm{kDa}$ was determined using a commercially available kit following the manufacturer's recommendations (Hyaluronan Quantikine ELISA, cata$\log$ DHYALO, R\&D Systems). The results were calculated as $\mathrm{ng} / \mathrm{mL}$ of the recovered BAL.

Adoptive transfer of alveolar macrophages. Mouse primary alveolar macrophages were isolated by BAL performed on euthanized mice with $3 \mathrm{~mL}$ PBS and $1 \mathrm{mM}$ EDTA. The lavage was centrifuged at $300 \mathrm{~g}$ for 10 minutes and resuspended in RPMI supplemented with 10\% FBS and plated at a density of 100,000 cells $/ \mathrm{cm}^{2}$. Alveolar macrophage purity was analyzed by flow cytometry and confirmed to be greater than $95 \%$. For the gating strategy, after gating out doublets using a forward scatter A (FSC-A) plot versus a FSC-H plot, nonimmune cells (CD45-), dead cells (eFluor $506^{+}$), and immune cell populations $\left(C D 45^{+}\right)$were

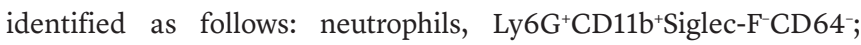
eosinophils, Siglec-F ${ }^{\text {int }}$ Ly6G ${ }^{-}$CD $11 b^{+}{ }^{+}$D 64 $4^{-}$; alveolar macrophages, $\mathrm{CD}^{+} 4^{+}$Siglec- $\mathrm{F}^{\mathrm{hi}}$; interstitial macrophages, CD $64^{+}$Siglec- $\mathrm{F}^{-} \mathrm{CD} 24^{-}$; classical monocytes, CD11b ${ }^{+} \mathrm{CD} 64-\mathrm{CD} 24-\mathrm{Ly}^{6} \mathrm{C}^{+}$; nonclassical mono-

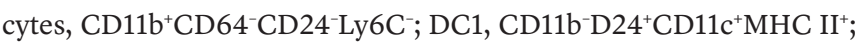
DC2, CD11 b ${ }^{+}$244 $4^{+} \mathrm{CD} 11 \mathrm{c}^{+} \mathrm{MHC} \mathrm{II}^{+}$; NK cells, CD64-NK1.1 ${ }^{+}$; CD4 ${ }^{+} \mathrm{T}$ cells, $\mathrm{CD}^{+} \mathrm{CD}^{+}$; and $\mathrm{CD} 8^{+} \mathrm{T}$ cells, $\mathrm{CD}^{+} \mathrm{CD} 8^{+}$. Alveolar macrophages were adoptively transferred into clodronate-pretreated mice immediately after sorting. Recipient mice were pretreated with $50 \mu \mathrm{L}$ clodronate-loaded liposomes (intratracheally) 72 hours before the adoptive transfer of donor alveolar macrophages to partially deplete TRAMs and make the niche permissive for engraftment. Donor alveolar macrophages $\left(1.5 \times 10^{5}\right.$ cells in $50 \mathrm{~mL}$ PBS $)$ were transferred via intratracheal instillation into isoflurane-anesthetized mice. CD45.1/.2 mice were used to discriminate between the recipient and donor cells.

In vitro bone marrow cell culture, bone marrow chimeras, and bone marrow chimeras with thoracic shielding. Bone marrow was isolated from the tibiae of 12-week-old C57BL/6 mice and incubated in RPMI 1640 medium supplemented with FBS, antibiotic, and $20 \mathrm{ng} /$ mL M-CSF for 5 days before transfer onto plates coated with low- or high-molecular-weight hyaluronan, laminin, collagen IV, or extracellular matrix from MLE-12 cell monolayers, where they were cultured for 3 more days in media supplemented with $20 \mathrm{ng} / \mathrm{mL}$ GM-CSF. Bone marrow chimeras were established by transferring $5 \times 10^{6}$ bone marrow cells isolated from C57BL/6 mice (this strain expresses CD45.2 alloantigen) into 8-week-old lethally irradiated (single dose of 1000 cGy $\gamma$-radiation using a Cs-137-based Gammacell 40 Irradiator, Nordion) recipient mice (expressing CD45.1 alloantigen). Mice were maintained on autoclaved water supplemented with antibiotics (trimethoprim/sulfamethoxazole, Hi-Tech Pharmacal) for 4 weeks after bone marrow transfer and then switched to a normal housing regimen. CD 45.2 to CD45.1 bone marrow chimeras were used for experiments 8 weeks after bone marrow transfer, at which time $>95 \%$ of all leukocytes and $100 \%$ of monocytes and neutrophils in peripheral blood were of donor origin. Bone marrow chimeras with thoracic shielding were used to assess the origin of pulmonary macrophages (TRAMs vs. MoAMs) and were generated in a manner similar to that previously described (8). Briefly, to protect TRAMs from radiation, we applied a uniform lead shield that covered the lungs during irradiation. To eliminate the residual recipient bone marrow in the shielded region, mice were treated with the myeloablative agent busulfan (30 $\mathrm{mg} / \mathrm{kg}$ body weight; MilliporeSigma) $(8,51)$ six hours after the irradiation, followed 12 hours later by bone marrow infusion. Chimerism was assessed 2 months after the procedure via FACS analysis of the peripheral blood collected from facial veins. Bone marrow chimeras with thoracic shielding were maintained on antibiotics for 4 week as described above and then switched back to the normal housing regimen. The following products and concentrations were used: M-CSF (catalog 416-ML/CF, R\&D Systems) and GM-CSF (catalog 130094-043, Miltenyi Biotec) at a final concentration of $5 \mu \mathrm{g} / \mathrm{mL}$; lowmolecular-weight hyalurone (catalog GLR001, R\&D Systems) at a final concentration of $5 \mu \mathrm{g} / \mathrm{cm}^{2}$; high-molecular-weight hyalurone (catalog GLR002, R\&D Systems) at a final concentration of $5 \mu \mathrm{g} / \mathrm{cm}^{2}$; collagen IV (catalog 354233, Corning) at a final concentration of 10 $\mu \mathrm{g} / \mathrm{cm}^{2}$; laminin A (catalog 354232, Corning) at a final concentration of $10 \mu \mathrm{g} / \mathrm{cm}^{2}$; RGD peptide (catalog TRUESRGD-1EA, MilliporeSigma) at a final concentration of $5 \mu \mathrm{g} / \mathrm{cm}^{2}$; scrambled peptide (catalog TRU-GD2, MilliporeSigma) at a final concentration of $5 \mu \mathrm{g} / \mathrm{cm}^{2}$; and RPMI (catalog 11875119, Gibco, Thermo Fisher Scientific).

Parabiosis. Parabiosis was performed as described previously (52). Briefly, parabiont mice were cohoused for 2 weeks prior to surgery. After anesthesia and preparation of the surgery site, the skin flap from elbow to knee was created on the left and right sides of the parabionts, and, accordingly, ankles and knees of the parabionts were joined using 

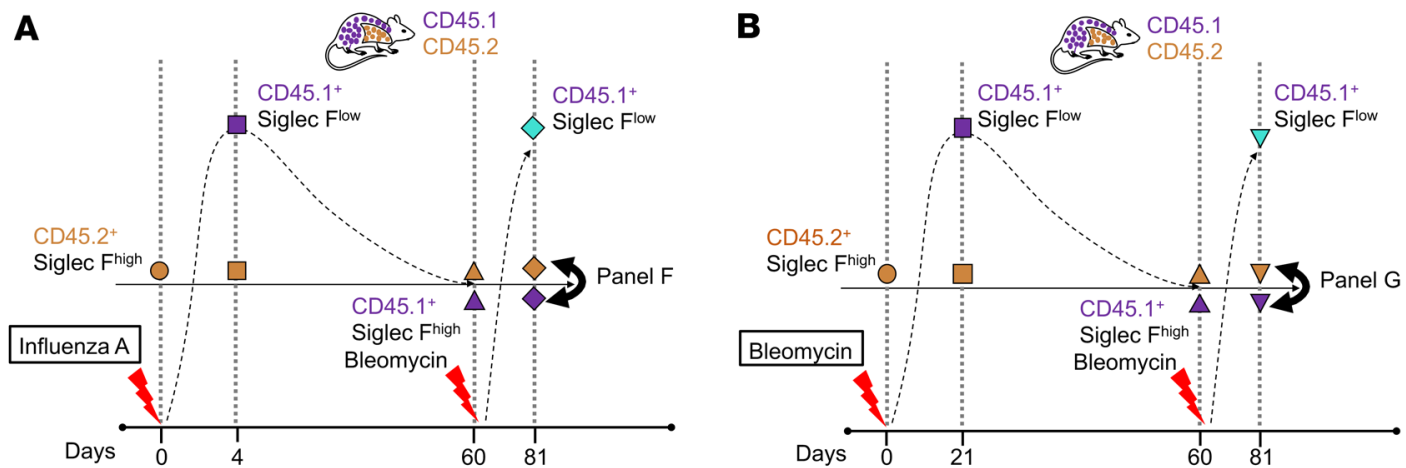

\section{Ontogeny (color) $\bigcirc$ TRAM MoAM $_{1} \bigcirc \mathrm{MOAM}_{2}$}

Time (shape) Before first injury $\square$ After first injury

\section{C}

\begin{tabular}{|c|c|c|c|}
\hline Population & Time (d) & Markers & Symbol(s) \\
\hline Tissue-resident alveolar macrophages & $\begin{array}{l}0(\mathrm{~T} 1), 4 \text { or } 21(\mathrm{~T} 2) \\
60(\mathrm{~T} 3), 81(\mathrm{~T} 4)\end{array}$ & CD45.2+SiglecFhigh & \\
\hline $\begin{array}{l}\text { Monocyte-derived alveolar macrophages recruited to the lung after } \\
\text { the first injury }\end{array}$ & $\begin{array}{l}4 \text { (influenza), } 21 \\
\text { (bleomycin) }\end{array}$ & CD45.1+SiglecFlow & \\
\hline $\begin{array}{l}\text { Monocyte-derived alveolar macrophages recruited to the lung after } \\
\text { the second injury }\end{array}$ & 81 & CD45.1+SiglecFlow & \\
\hline MoAMs persisting after first injury & 60 & CD45.1+SiglecFhigh & \\
\hline MoAMs persisting after first injury responding to second injury & 81 & CD45.1+SiglecFigh & \\
\hline
\end{tabular}

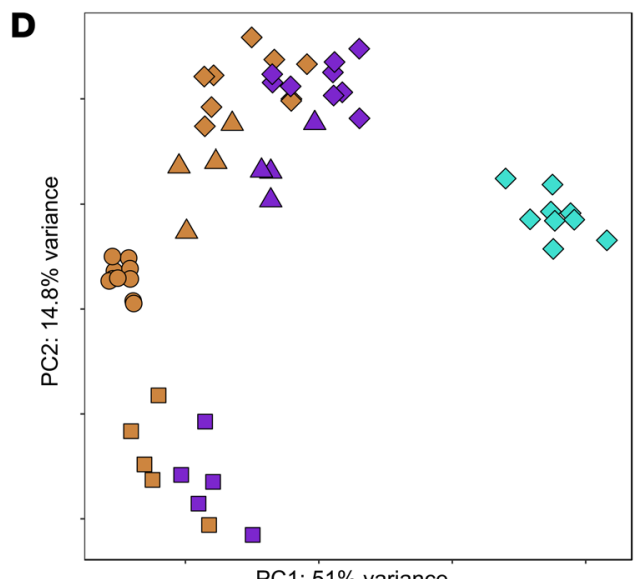

PC1: $51 \%$ variance

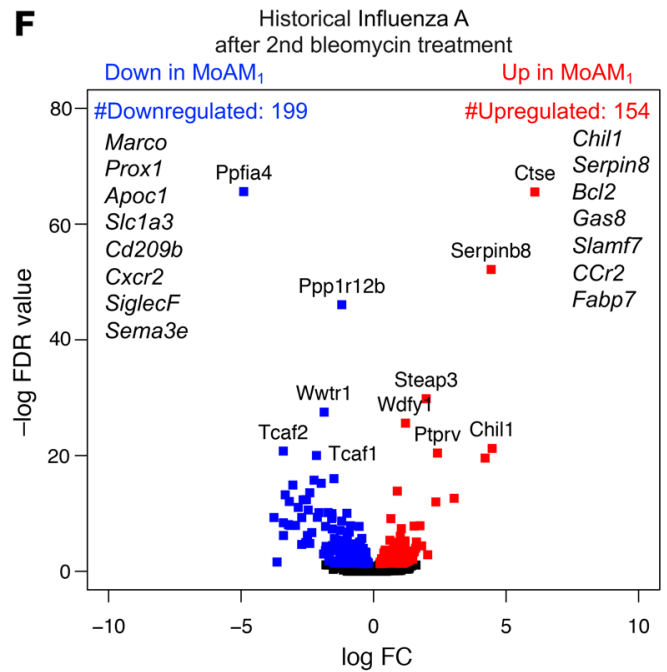

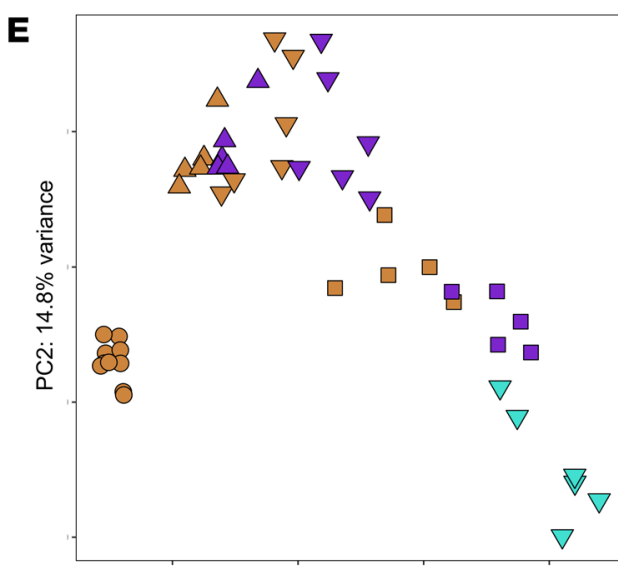

PC1: $51 \%$ variance

G

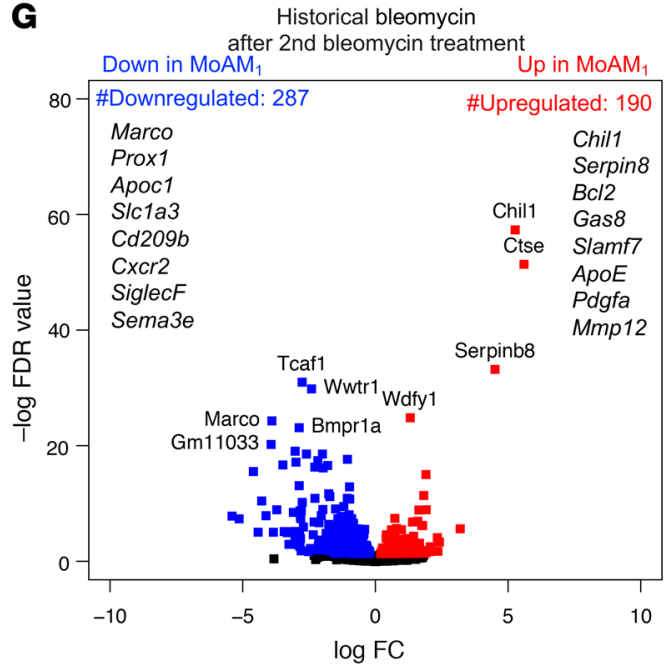


Figure 8. The response of TRAMs and newly resident MoAMs to a second challenge is similar. (A) Experimental design for the PCA data in $\mathbf{D}$. Mice were intratracheally infected with influenza $A$ virus on day 0 followed by treatment with bleomycin on day 60. (B) Experimental design for the PCA data in E. Mice were administered intratracheal bleomycin on day 0 followed by treatment with a second dose of bleomycin on day 60. (C) Description of cell populations subjected to RNA-Seq. (D) PCA of alveolar macrophage transcriptomes. Colors and symbols refer to panels $\mathbf{A}$ and $\mathbf{C}$. (E) PCA of alveolar macrophage transcriptomes. Colors and symbols refer to panels $\mathbf{B}$ and $\mathbf{C}$. (F) Volcano plot shows differentially expressed genes $($ FDR $<0.05)$ between TRAMs and MoAMs (recruited in response to historic influenza $A$ virus-induced pneumonia as the first injury; black double arrow in $\mathbf{A}$ ) after the second injury with bleomycin. Representative genes are shown adjacent to the plot (see Supplemental Table 18 for the full list of genes). (G) Volcano plot shows differentially expressed genes (FDR < 0.05 ) between TRAMs and MoAMs (recruited in response to bleomycin exposure as the first injury; black double arrow in B) after the second injury with bleomycin. Representative genes are shown adjacent to the plot (see Supplemental Table 19 for the full list of genes).

nonabsorbable suture, and skin flaps were joined using continuous uninterrupted suture. Mice were treated with Buprenorphine SR (Covetrus) during the first 2 weeks of recovery and provided recovery diet gel $\left(\mathrm{ClearH}_{2} \mathrm{O}\right)$. After recovery, mice were switched back to the normal housing regimen.

Tissue preparation and flow cytometry. Tissue preparation for flow cytometric analysis and cell sorting was performed as previously described (8). Blood was collected into EDTA-containing tubes via facial vein bleeding (from live animals) or cardiac puncture (from euthanized animals). Whole blood was stained with fluorochrome-conjugated antibodies, and erythrocytes were then lysed using BD FACS lysing solution. For single-cell suspensions obtained from tissues, erythrocytes were lysed using BD Pharm Lyse, and cells were counted using the Nexcelom K2 Cellometer C automated cell counter with acridine orange (AO) and propidium iodide (PI) reagent. Cells were stained with eFluor 506 (eBioscience) viability dyes, incubated with BD Fc Block, and stained with fluorochrome-conjugated antibodies (see Methods above). Data acquisition and cell sorting were performed at the Northwestern University RLHCCC Flow Cytometry core facility on BD SORP FACS Aria III, BD LSR II, BD Fortessa, and BD Symphony instruments. Sorting was performed using a $100 \mu \mathrm{m}$ nozzle and 40 psi pressure. Compensation, analysis, and visualization of the flow cytometric data were performed using FlowJo software (Tree Star). "Fluorescence minus one" controls were used when necessary to set up gates.

Transcriptome profiling via RNA-Seq. Flow cytometric sorting was used to isolate mouse alveolar macrophages and AT2 cells at the indicated time points for each experiment. Cells were sorted with MACS buffer, pelleted, and lysed in RLT Plus buffer supplemented with 2-mercaptoethanol (QIAGEN). The RNeasy Plus Mini Kit (QIAGEN) was used to isolate RNA and remove genomic DNA. RNA quality was assessed with the 4200 TapeStation System (Agilent Technologies). Samples with an RNA integrity number (RIN) lower than 7 were discarded. RNA-Seq libraries were prepared from 100 ng total RNA using the NEB Next RNA Ultra Kit (QIAGEN) with poly(A) enrichment. Libraries were quantified and assessed using the Qubit Fluorimeter (Invitrogen, Thermo Fisher Scientific) and the Agilent TapeStation 4200. Libraries were sequenced on the NextSeq 500 instrument (Illumina) at 75 bp length, single-end reads.
The average reading depth across all experiments exceeded $6 \times 10^{6}$ per sample, and over $94 \%$ of the reads had a Q score above 30 . For RNA-Seq analysis, reads were demultiplexed using bcl2fastq (version 2.17.1.14). Read quality was assessed with FastQC. Samples that did not pass half of the 12 assessed quality control (QC) statistics were eliminated. Low-quality base calls were trimmed using Trimmomatic (version 0.33). Reads were then aligned to the Mus musculus reference genome (with $\mathrm{mm} 10$ assembly) using the TopHat2 aligner (version 2.1.0). Count tables were generated using HTSeq (version 0.6.1). Raw counts were processed in $\mathrm{R}$ (version 3.4.4) using edge R (version 3.20.9) to generate normalized counts (53). Negative binomial likelihood with the default setting followed by generalized linear models fitting were used to estimate differentially expressed genes. FDR $q$ values were used to correct for multiple comparisons, and a value of 0.05 was used as a threshold for statistical significance. $\mathrm{K}$-means clustering was performed using the built-in R Stats Package (version 3.4.4). GO analysis was performed using GOrilla (54) on 2 unranked gene lists. The RNA-Seq data sets are available at in the NCBI's Gene Expression Omnibus (GEO) database (GEO GSE134397). Computationally intensive work was performed on Northwestern University's Quest High-Performance Computing Cluster (Northwestern IT and Research Computing).

Single-cell RNA-Seq. Single-cell suspensions were prepared as described above with slight modification. Mice were euthanized with sodium pentobarbital. The chest cavity was opened and lungs were perfused through the right ventricle with $10 \mathrm{~mL}$ HBSS. The lungs were removed and, using a $30 \mathrm{G}$ needle, infused with $1 \mathrm{~mL}$ dispase (Corning) with DNase I (MilliporeSigma). Lungs were incubated at room temperature with gentle agitation for 45 minutes, followed by gentle teasing using forceps into small (1-2 $\mathrm{mm}$ ) fragments, followed by incubation in digestion buffer for another 15 minutes. The resulting suspension was passed through a $70 \mu \mathrm{m}$ cell strainer (Falcon), washed with DMEM (Corning) supplemented with 5\% FBS (Corning), and pelleted by centrifugation, and erythrocytes were lysed using BD Pharm Lyse (BD Biosciences). The resulting single-cell suspension was kept in DMEM and FBS and passed twice through $40 \mu \mathrm{m}$ cell strainer (Falcon). Cells were counted using a Cellometer K2 (Nexcelom) with the nucleic acid binding dyes AO, to calculate the total number of nucleated cells, and PI, to count dead cells (cell viability exceeded 85\%). All manipulations were performed using wide-bore tips (Axygen). Single-cell 3' RNA-Seq libraries were prepared using the Chromium Single Cell V2 Reagent Kit and Controller (10x Genomics). Libraries were assessed for quality (TapeStation 4200) and then sequenced on a HiSeq 4000 instrument (Illumina) for single-cell RNA-Seq libraries. Initial data processing was performed using the Cell Ranger, version 2.0 pipeline (10× Genomics), and reads were mapped to the $\mathrm{mm} 10$ version of the mouse genome (Ensemble build 84). Downstream single-cell RNA-Seq analysis was performed using the Seurat Package (version 3.1.0) following the standard workflow posted on the Satija Laboratory website (https:// satijalab.org/seurat/) (55). Specifically, SCTransform with an anchorbased integration approach was used to integrate 6 different public data sets. The pseudo-bulk RNA-Seq count matrix was generated by averaging the counts for gene expression in all alveolar macrophages from each individual. The detailed methods and codes are available on GitHub (https://github.com/NUPulmonary/Doublehit_Human_ scRNA_Analysis; branch - master; commit ID: e486203eb0e1437d73be589a31c803fbc46182bd). 
A

$\begin{array}{ll}\text { Ontogeny (color) } & \text { Time (shape) } \\ \text { TRAM } & \text { Before first injury } \\ \text { MoAM }_{1} & \text { After first injury } \\ \text { MoAM }_{2} & \text { Before second injury } \\ & \text { After second injury } \\ \text { (historic bleomycin) } \\ \text { After second injury } \\ \text { (historic influenza A) }\end{array}$

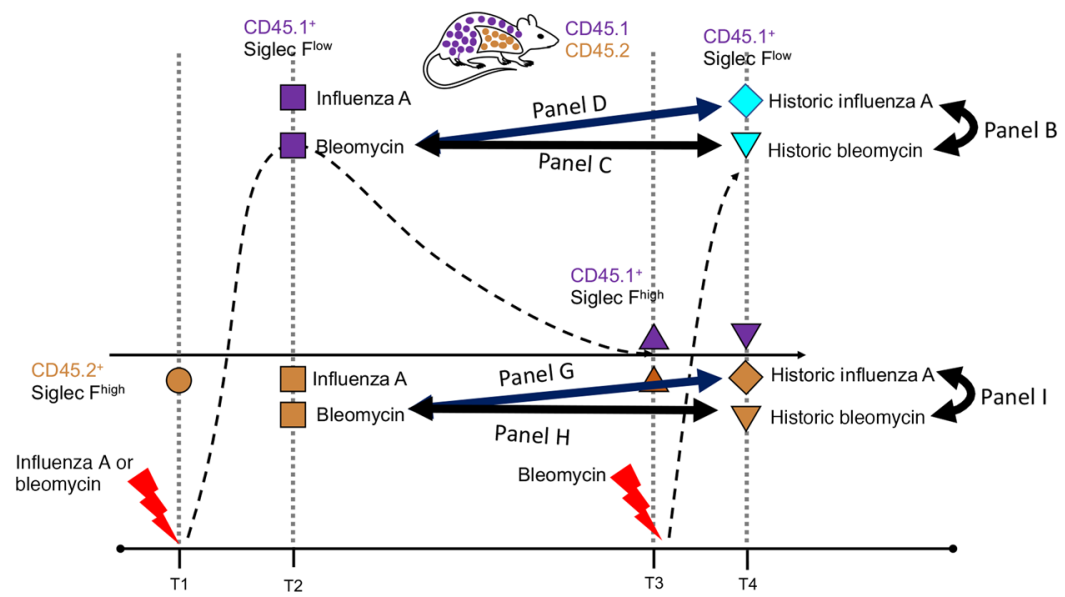

F

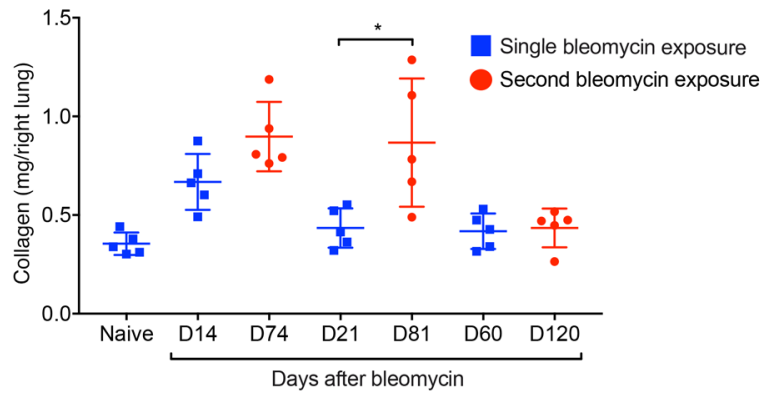

G

\section{C}

Cell cycle

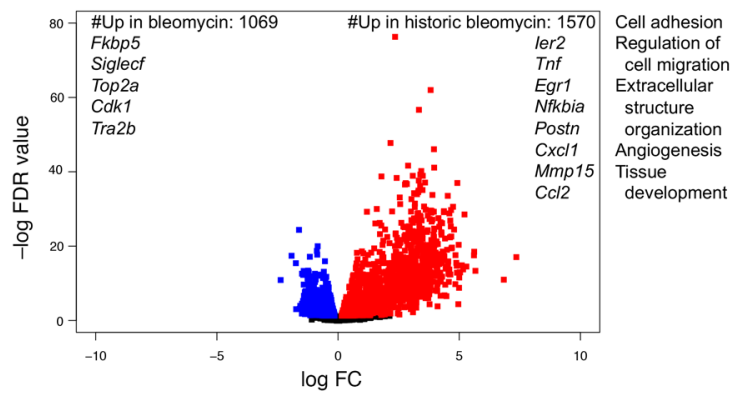

D

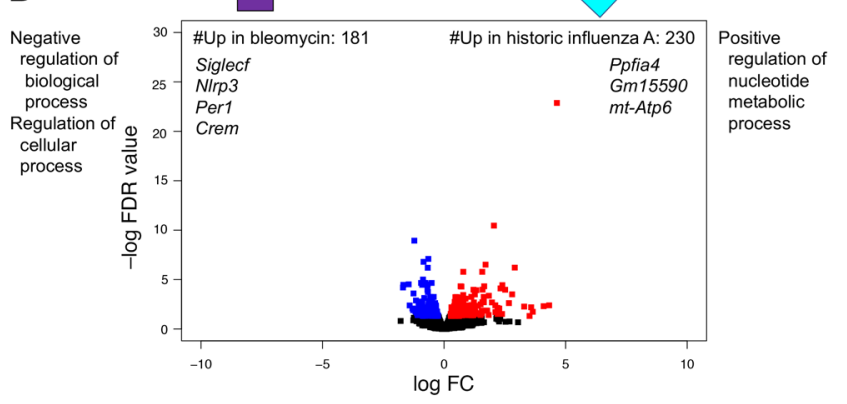

E

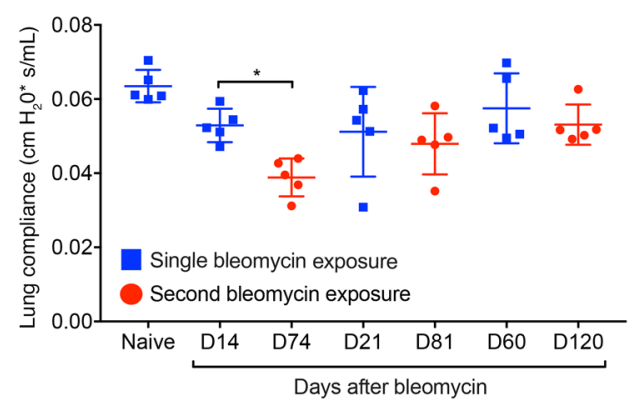

$\underset{\text { transduction }}{\text { Signal }}$

Inflammatory

response

Regulation of

cell motility

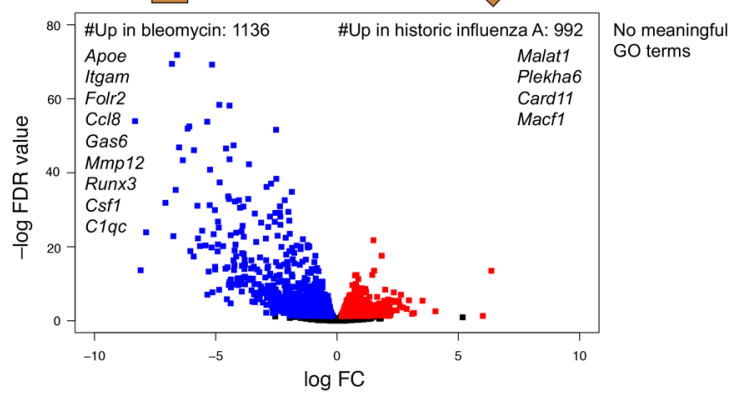

H

Response to externa biotic

Stimulus
Regulation of

cell migration

Response to

stress

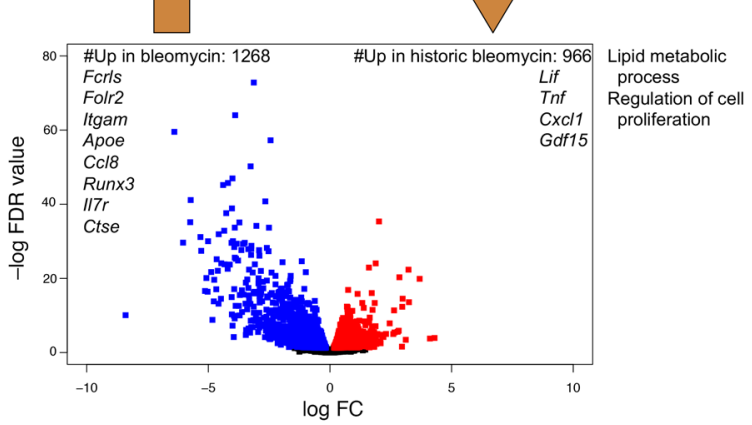

I

Cell cycle

process

organization

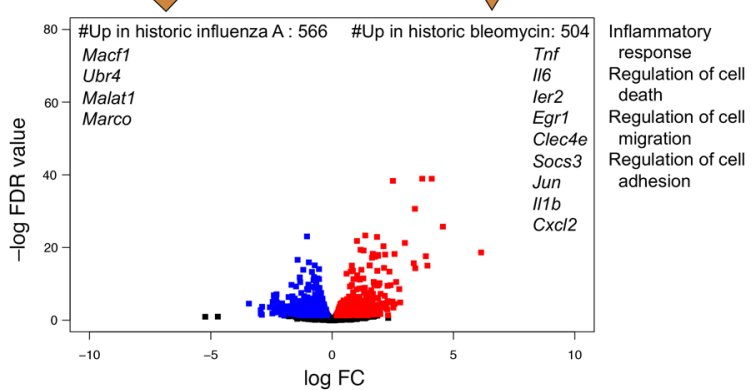


Figure 9. The environment determines the response of resident and recruited alveolar macrophages after repeated injury. (A) Schematic for the experimental design for panels B-I. Pairwise comparisons described in panels B-G and in panels $\mathbf{G}-\mathbf{I}$ are indicated by the double black arrows. (B) Volcano plot shows differentially expressed genes between MoAMs recruited after bleomycin exposure in mice historically treated with bleomycin and mice historically infected with influenza $A(F D R<0.05)$ (see Supplemental Table 24 for the full list of genes). (C) Volcano plot shows differentially expressed genes between MoAMs recruited after bleomycin exposure in untreated mice and mice historically exposed to bleomycin (FDR $q<0.05$ ). (D) Volcano plot shows differentially expressed genes between MoAMs recruited after bleomycin exposure in untreated mice and mice historically infected with influenza A virus (FDR $q<0.05$ ). (E) Lung compliance was measured in mice after a single bleomycin exposure and 2 sequential bleomycin exposures separated by 60 days. ${ }^{*} P<0.05$, 2-way ANOVA followed by unpaired Student's $t$ test, for comparison between the first and second bleomycin exposures. $n=5$ mice per group. (F) Collagen levels were measured in mice after a single bleomycin exposure and 2 sequential bleomycin exposures separated by 60 days. ${ }^{*} P<0.05$, 2-way ANOVA followed by an unpaired $t$ test, for comparison between first and second bleomycin exposures. $n=5$ mice per group. (C) Volcano plot shows differentially expressed genes in TRAMs after bleomycin exposure in untreated mice and mice historically infected with influenza A virus (FDR $<0.05)$. (H) Volcano plot shows differentially expressed genes in TRAMs after bleomycin exposure in untreated mice and mice historically exposed to bleomycin (FDR < 0.05). (I) Volcano plot shows differentially expressed genes in TRAMs after bleomycin exposure in mice historically exposed to influenza A virus and mice historically exposed to bleomycin (FDR $<0.05$ ). See also Supplemental Table 23. D, day.

Modified reduced representation bisulfite sequencing. Measurement and analysis of DNA methylation were performed as previously described (40). Briefly, genomic DNA was isolated from sorted macrophage populations (AllPrep Micro Kit, QIAGEN). Endonuclease digestion, size selection, bisulfite conversion, and library preparation were prepared as previously described from approximately $250 \mathrm{ng}$ input genomic DNA. Bisulfite conversion efficiency was $99.6 \%$ as estimated by the measured percentage of unmethylated CpGs in $\lambda$-bacteriophage DNA (N3013S, New England BioLabs) spiked into each sample. Equimolar ratios of 4 libraries per run were sequenced on the NextSeq 500 Sequencer (Illumina), at 75 bp length, and single-end reads were sequenced using the NextSeq 500/550 V2 High Output Reagent Kit. Demultiplexing, trimming, alignment to the Mus musculus reference genome (with mm10 assembly), and methylation calling were performed as previously described (40). Quantification was performed using the DSS R/Bioconductor package (56) and the SeqMonk platform (57). The RRBS data set is available in the NCBI's GEO database (GEO GSE134238). Computationally intensive work was performed on Northwestern University's Quest High-Performance Computing Cluster (Northwestern IT and Research Computing).

Data and software availability. The RNA-Seq data sets, containing raw and processed data, are available in the NCBI's GEO database (GEO GSE134238, RRBS data set and GSE134397, RNA-Seq data set). The $\mathrm{R}$ code program used for the analysis is publicly available on GitHub (https://github.com/NUPulmonary/Doublehit_Human_ scRNA_Analysis).

Statistics. Data are reported as the mean \pm SEM and subjected to 1-way ANOVA. For pairwise significance, a 2-tailed Student's $t$ test with Benjamini-Hochberg correction for multiple comparisons was applied (R stats, version 3.4.4; GraphPad Prism 7, GraphPad Software). Statistical methods for RNA-Seq are described above. Sample power and size were estimated on the basis of our previous work and published articles. The observed data met or exceeded the required sample-size criteria. The statistical parameters and criteria for significance are described in the figure legends.

Study approval. This study was approval by the IRB of Northwestern University (approval number IS00001035), and all animal experiments and procedures were performed according to protocols approved by the IACUC of Northwestern University.

\section{Author contributions}

ZR contributed to the conceptualization, methodology, software development, validation, formal analysis, investigation, data curation, writing the manuscript, visualization, and supervision of the study. ACMP contributed to the methodology, formal analysis, investigation, data curation, and image visualization. NJ contributed to the methodology, formal analysis, investigation, and image visualization. SW contributed to the methodology, formal data analysis and curation, and image visualization. TS contributed to the methodology, validation, formal analysis, data curation, and investigation. MC and ZL contributed to the methodology, investigation, formal analysis, and data curation. LS and RPA contributed to methodology, formal analysis, and data curation. CIC contributed to the methodology, data validation, and investigation. SS and ZC contributed to investigation, resources, formal data analysis, methodology, data validation, and image visualization. PAR, JMW, and KRA contributed to investigation, resources, and analysis. JMD and KAH contributed to the methodology, investigation, and data curation. CER contributed to the methodology and investigation. HAV contributed to the methodology, software development, validation, formal analysis, and data curation. KN contributed to the methodology and investigation. AYM contributed to the methodology, formal analysis, data curation, and image visualization. DRW contributed to the methodology, software development, validation, formal analysis, data curation, writing of the manuscript, and image visualization. RIM and GMM contributed to the conceptualization, data curation, and supervision. $\mathrm{AB}, \mathrm{CJG}, \mathrm{KMR}$, and NSC provided resources and contributed to the conceptualization, methodology, validation, formal analysis, investigation, data curation, writing of the manuscript, image visualization, supervision, and project administration. JIS provided resources and contributed to the conceptualization, data curation, writing of the manuscript, supervision, and project administration. WEB contributed to the methodology and provided resources. BDS provided resources and contributed to conceptualization, methodology, validation, formal analysis, investigation, and data curation. HP, AVM, and GRSB provided resources and contributed to the conceptualization, methodology, validation, formal analysis, investigation, data curation, writing of the manuscript, image visualization, study supervision, project administration, and funding acquisition. The order of the co-first authors was determined alphabetically by first name.

\section{Acknowledgments}

The Northwestern University Flow Cytometry Core Facility is supported by a National Cancer Institute (NCI) Cancer Center 
Support Grant (P30 CA060553, awarded to the Robert H. Lurie Comprehensive Cancer Center). Cell sorting was performed on a BD FACSAria SORP cell sorter purchased through the support of NIH grant 1S10OD011996-01. This research was supported in part through the computational resources and staff contributions provided by the Genomics Compute Cluster, which is jointly supported by the Feinberg School of Medicine, the Center for Genetic Medicine, and the Feinberg School of Medicine's Department of Biochemistry and Molecular Genetics, the Office of the Provost, the Office for Research, and Northwestern Information Technology. GRSB is supported by the NIH (U19AI135964，P01AG049665，R01HL147575，P01HL071643, and R01HL154686) and the Department of Veterans Affairs (I01CX001777). BDS is supported by the NIH (K08HL128867, U19AI135964, R01HL149883, and P01AG049665). PAR is supported by the NIH (K08HL146943) and by an ATS Foundation/ Boehringer Ingelheim Partner Award for research in idiopathic pulmonary fibrosis. AVM is supported by the NIH (U19AI135964, P01AG049665, R56HL135124, and R01HL153312). AB is supported by the NIH (HL145478, HL147290, and HL147575). JIS is supported by the NIH (R01HL154686, P01AG049665, and P01HL071643). RGW is supported by the NIH (U19AI135964) and a GlaxoSmithKline Distinguished Scholar in Respiratory Health grant from the CHEST Foundation. NSC is supported by the NIH (P01AG049665 and P01HL071643). DRW is supported by funding from the Arthritis National Research Foundation (ANRF), the American Heart Association (AHA), the Scleroderma Foundation, the American Lung Association (ALA), the American Thoracic Society (ATS), the American Federation for Aging (AFAR), and the NIH (U19AI135964). SW is supported by the MSD Life Science Foundation, the Public Interest Incorporated Foundation, Japan, and by a David W. Cugell and Christina Enroth-Cugell Fellowship. KMR is supported by the NIH (P01AG049665 and P01HL071643). CJG is supported by the NIH (R01AR073270 and R01HL134800). GMM is supported by the NIH (R01ES015024, U01ES026718, and P3OES027792). TS is supported by the NIH (K99AG068544) and the National Science Foundation (NSF) (1956338). ZR is supported by the AHA (2OPOST35180141).

Address correspondence: G.R. Scott Budinger or Alexander V. Misharin, Pulmonary and Critical Care Medicine, Northwestern University, 240 E Huron Street, M300, Chicago, Illinois 60611, USA. Phone: 312.908.8163; Email: s-buding@northwestern.edu (GRSB); Email: a-misharin@northwestern.edu (AVM).
1. Zhou F, et al. Clinical course and risk factors for mortality of adult inpatients with COVID-19 in Wuhan, China: a retrospective cohort study. Lancet. 2020;395(10229):1054-1062.

2. Budinger GRS, et al. The intersection of aging biology and the pathobiology of lung diseases: a joint NHLBI/NIA workshop. J Gerontol A Biol Sci Med Sci. 2017;72(11):1492-1500.

3. Hussell T, Bell TJ. Alveolar macrophages: plasticity in a tissue-specific context. Nat Rev Immunol. 2014;14(2):81-93.

4. Yona S, et al. Fate mapping reveals origins and dynamics of monocytes and tissue macrophages under homeostasis. Immunity. 2013;38(1):79-91.

5. Hashimoto D, et al. Tissue-resident macrophages self-maintain locally throughout adult life with minimal contribution from circulating monocytes. Immunity. 2013;38(4):792-804.

6. Guilliams M, et al. Alveolar macrophages develop from fetal monocytes that differentiate into longlived cells in the first week of life via GM-CSF. J Exp Med. 2013;210(10):1977-1992.

7. Lavin Y, et al. Tissue-resident macrophage enhancer landscapes are shaped by the local microenvironment. Cell. 2014;159(6):1312-1326.

8. Misharin AV, et al. Monocyte-derived alveolar macrophages drive lung fibrosis and persist in the lung over the life span. JExp Med. 2017;214(8):2387-2404.

9. Horvath S, Raj K. DNA methylation-based biomarkers and the epigenetic clock theory of ageing. Nat Rev Genet. 2018;19(6):371-384.

10. Morales-Nebreda L, et al. Aging imparts cell-autonomous dysfunction to regulatory $\mathrm{T}$ cells during recovery from influenza pneumonia [preprint]. https://doi. org/10.1101/2020.06.05.135194. Posted on bioRxiv June 05, 2020.

11. Sakai M, et al. Liver-derived signals sequentially reprogram myeloid enhancers to initiate and maintain kupffer cell identity. Immunity. 2019;51(4):655-670.e8.

12. Wong CK, et al. Aging impairs alveolar macrophage phagocytosis and increases influenza-induced mortality in mice. J Immunol. 2017;199(3):1060-1068.

13. Happle C, et al. Pulmonary transplantation of macrophage progenitors as effective and long-lasting therapy for hereditary pulmonary alveolar proteinosis. Sci Transl Med. 2014;6(250):250ra113.

14. Suzuki T, et al. Pulmonary macrophage transplantation therapy. Nature. 2014;514(7523):450-454

15. Angelidis I, et al. An atlas of the aging lung mapped by single cell transcriptomics and deep tissue proteomics. Nat Commun. 2019;10(1):963.

16. Schaum N, et al. Single-cell transcriptomics of 20 mouse organs creates a Tabula Muris. Nature. 2018;562(7727):367-372.

17. Reyfman PA, et al. Single-cell transcriptomic analysis of human lung provides insights into the pathobiology of pulmonary fibrosis. Am J Respir Crit Care Med. 2019;199(12):1517-1536.

18. Madissoon E, et al. scRNA-seq assessment of the human lung, spleen, and esophagus tissue stability after cold preservation. Genome Biol. 2019;21(1):1.

19. Raredon MSB, et al. Single-cell connectomic analysis of adult mammalian lungs. Sci Adv. 2019;5(12):eaaw3851.

20. Morse C, et al. Proliferating SPP1/MERTKexpressing macrophages in idiopathic pulmonary fibrosis. Eur Respir J. 2019;54(2):1802441.

21. Valenzi E, et al. Single-cell analysis reveals fibroblast heterogeneity and myofibroblasts in systemic sclerosis-associated interstitial lung disease. Ann Rheum Dis. 2019;78(10):1379-1387.

22. Habermann AC, et al. Single-cell RNA sequencing reveals profibrotic roles of distinct epithelial and mesenchymal lineages in pulmonary fibrosis. Sci Adv. 2020;6(28):eaba1972.

23. Conboy MJ, et al. Heterochronic parabiosis: historical perspective and methodological considerations for studies of aging and longevity. Aging Cell. 2013;12(3):525-530.

24. Suzuki T, et al. Increased pulmonary GM-CSF causes alveolar macrophage accumulation: mechanistic implications for desquamative interstitial pneumonitis. Am J Respir Cell Mol Biol. 2019;62(1):87-94.

25 . Huang $\mathrm{H}$, et al. Protective effects of recombinant human granulocyte macrophage colony stimulating factor on H1N1 influenza virus-induced pneumonia in mice. Cytokine. 2010;51(2):151-157.

26. Huang F-F, et al. GM-CSF in the lung protects against lethal influenza infection. Am J Respir Crit Care Med. 2011;184(2):259-268.

27. Herold S, et al. Inhaled granulocyte/macrophage colony-stimulating factor as treatment of pneumonia-associated acute respiratory distress syndrome. Am J Respir Crit Care Med. 2014;189(5):609-611.

28. Runyan CE, et al. Impaired phagocytic function in CX3CR1 $1^{+}$tissue-resident skeletal muscle macrophages prevents muscle recovery after influenza A virus-induced pneumonia in old mice. Aging Cell. 2020;19(9):e13180.

29. Budinger GR, Sznajder JI. The alveolar-epithelial barrier: a target for potential therapy. Clin Chest Med. 2006;27(4):655-669.

30. Skelly DA, et al. Single-cell transcriptional profiling reveals cellular diversity and intercommunication in the mouse heart. Cell Rep. 2018;22(3):600-610.

31. Ramilowski JA, et al. A draft network of ligandreceptor-mediated multicellular signalling in human. Nat Commun. 2015;6:7866.

32. Schiller HB, et al. Time- and compartmentresolved proteome profiling of the extracellular 
niche in lung injury and repair. Mol Syst Biol. 2015;11(7):819.

33. Bell TJ, et al. Defective lung function following influenza virus is due to prolonged, reversible hyaluronan synthesis. Matrix Biol. 2019;80:14-28.

34. Dong Y, et al. The survival of fetal and bone marrow monocyte-derived alveolar macrophages is promoted by CD44 and its interaction with hyaluronan. Mucosal Immunol. 2018;11(3):601-614.

35. van de Laar L, et al. Yolk sac macrophages, fetal liver, and adult monocytes can colonize an empty niche and develop into functional tissue-resident macrophages. Immunity. 2016;44(4):755-768.

36. Chiarella SE, et al. $\beta 2$-Adrenergic agonists augment air pollution-induced IL-6 release and thrombosis. J Clin Invest. 2014;124(7):2935-2946.

37. Soberanes S, et al. Metformin targets mitochondrial electron transport to reduce air-pollution-induced thrombosis. Cell Metab. 2019;29(2):335-347.e5.

38. McCubbrey AL, et al. Deletion of c-FLIP from $\mathrm{CD} 11 \mathrm{~b}^{\text {hi }}$ macrophages prevents development of bleomycin-induced lung fibrosis. Am J Respir Cell Mol Biol. 2018;58(1):66-78.

39. Gibbings SL, et al. Transcriptome analysis highlights the conserved difference between embryonic and postnatal-derived alveolar macrophages. Blood. 2015;126(11):1357-1366.

40. Weinberg SE, et al. Mitochondrial complex III is essential for suppressive function of regulatory T cells. Nature. 2019;565(7740):495-499.

41. Mould KJ, et al. Cell origin dictates programming of resident versus recruited macrophages during acute lung injury. Am J Respir Cell Mol Biol. 2017;57(3):294-306.

42. Degryse AL, et al. Repetitive intratracheal bleomycin models several features of idiopathic pulmonary fibrosis. Am J Physiol Lung Cell Mol Physiol. 2010;299(4):L442-L452.

43. Ortiz JR, et al. Population-based incidence estimates of influenza-associated respiratory failure hospitalizations, 2003 to 2009. Am J Respir Crit Care Med. 2013;188(6):710-715.

44. Jain S, et al. Community-acquired pneumonia requiring hospitalization among U.S. adults. N Engl JMed. 2015;373(5):415-427.

45. Chen CS, et al. Geometric control of cell life and death. Science. 1997;276(5317):1425-1428.

46. Ross FP, Teitelbaum SL. $\alpha v \beta 3$ and macrophage colony-stimulating factor: partners in osteoclast biology. Immunol Rev. 2005;208(1):88-105.

47. Netea MG, et al. Trained immunity: A program of innate immune memory in health and disease. Science. 2016;352(6284):aaf1098.

48. Yao Y, et al. Induction of autonomous memory alveolar macrophages requires $\mathrm{T}$ cell help and is critical to trained immunity. Cell. 2018;175(6):1634-1650.e17.

49. Lee J-H, et al. Surfactant protein-C chromatinbound green fluorescence protein reporter mice reveal heterogeneity of surfactant protein C-expressing lung cells. Am J Respir Cell Mol Biol. 2013;48(3):288-298.

50. Maus U, et al. Monocytes recruited into the alveolar air space of mice show a monocytic phenotype but upregulate CD14. Am J Physiol Lung Cell Mol Physiol. 2001;280(1):L58-L68.

51. Chevaleyre J, et al. Busulfan administration flexibility increases the applicability of scid repopulating cell assay in NSG mouse model. PLoS One. 2013;8(9):e74361.

52. Hsiao HM, et al. Spleen-derived classical monocytes mediate lung ischemia-reperfusion injury through IL-1 $\beta$. JClin Invest. 2018;128(7):2833-2847.

53. Robinson MD, et al. edgeR: a Bioconductor package for differential expression analysis of digital gene expression data. Bioinformatics. 2010;26(1):139-140.

54. Eden E, et al. GOrilla: a tool for discovery and visualization of enriched GO terms in ranked gene lists. BMC Bioinformatics. 2009;10:48.

55. Satija R, et al. Spatial reconstruction of singlecell gene expression data. Nat Biotechnol. 2015;33(5):495-502.

56. Feng H, et al. A Bayesian hierarchical model to detect differentially methylated loci from single nucleotide resolution sequencing data. Nucleic Acids Res. 2014;42(8):e69.

57. SeqMonk. Version 1.47.1. Andrews S; 2020. Accessed 19 Apr 2018. https://www.bioinformatics.babraham.ac.uk/projects/seqmonk/. 